Dieta humana, movilidad y tecnología en un contexto mortuorio del valle del Atuel: el registro de Cañada Seca-1 (San Rafael, Mendoza) Eva Ailén Peralta, María de la Paz Pompei, José Manuel López, Danae Fiore, Sergio Diéguez, Andrew Ugan, Adolfo F. Gil, Gustavo A. Neme Relaciones, 46(2), e020, julio-diciembre 2021 ISSN 1852-1479 | https://doi.org/10.24215/18521479e020 https://revistas.unlp.edu.ar/relaciones ISSN 0325-2221 (versión impresa) Sociedad Argentina de Antropología (SAA)

Buenos Aires I Argentina

\title{
DIETA HUMANA, MOVILIDAD Y TECNOLOGÍA EN UN CONTEXTO MORTUORIO DEL VALLE DEL ATUEL: EL REGISTRO DE CAÑADA SECA-1 (SAN RAFAEL, MENDOZA)
}

\author{
Eva Ailén Peralta*, María de la Paz Pompei**, José Manuel López,**, Danae Fiore ${ }^{* * * *}$, \\ Sergio Diéguez, ${ }^{* * * * *}$, Andrew Ugan ${ }^{* * * * * *}$, Adolfo F. Gil ${ }^{* * * * * * *}$, Gustavo A. Neme ${ }^{* * * * * * * * *}$
}

Fecha de recepción: 15 de enero de 2021

Fecha de aceptación: 9 de septiembre de 2021

\section{RESUMEN}

Este trabajo tiene como objetivo caracterizar el sitio Cañada Seca-1, un entierro múltiple $(N M I=24)$ localizado en el valle del Atuel y fechado en ca. 1500 años AP, a través de la integración de diferentes líneas de evidencia. A partir de este registro se discuten las interpretaciones en torno a la presencia de cultígenos y sus implicancias para la subsistencia humana en un

\footnotetext{
* Consejo Nacional de Investigaciones Científicas y Técnicas, Instituto de Evolución Ecología Histórica y Ambiente. E-mail: evaailenperalta@gmail.com

${ }^{* *}$ Consejo Nacional de Investigaciones Científicas y Técnicas, Instituto Superior de Estudios Sociales Universidad Nacional de Tucumán. E-mail: paz.pompei@gmail.com

${ }^{* * *}$ Consejo Nacional de Investigaciones Científicas y Técnicas, Instituto Argentino de Investigaciones de las Zonas Áridas - Facultad de Filosofía y Letras, Universidad Nacional de Cuyo. E-mail: mlopez@mendozaconicet.gob.ar

**** Consejo Nacional de Investigaciones Científicas y Técnicas, Asociación de Investigaciones Antropológicas - Universidad de Buenos Aires. E- mail: danae_fiore@yahoo.es

***** Comisión Nacional de Energía Atómica. E-mail: dieguez@cnea.gov.ar

******* Deparment of Anthropology, University of Utah. E-mail: andrew.ugan@gmail.com

******* Consejo Nacional de Investigaciones Científicas y Técnicas, Instituto de Evolución Ecología Histórica y Ambiente - Universidad Nacional de Cuyo. E-mail: agil@mendoza-conicet.gob.ar ${ }_{* * * * * * * *}$ Consejo Nacional de Investigaciones Científicas y Técnicas, Instituto de Evolución Ecología Histórica y Ambiente. E-mail: gneme@ mendoza-conicet.gob.ar
} 
área caracterizada tradicionalmente como el límite de la dispersión agrícola sudamericana, ca. 2200 años AP. Los resultados destacan que los individuos inhumados en el sitio no habrían desarrollado una estrategia fundamentalmente agrícola, aunque el maíz formó parte de su dieta. Además, la nueva información sobre los comportamientos mortuorios en la zona del valle del Atuel, ofrece evidencias tanto sobre movilidad como sobre el uso de tecnologías utilitarias (líticas) y no utilitarias (ornamentos líticos y malacológicos) que trascendieron la vida cotidiana y los acompañaron en la muerte.

Palabras clave: agricultura prehispánica - dieta-movilidad-isótopos estables - Mendoza

\section{HUMAN DIET, MOBILITY AND TECHNOLOGY IN A MORTUARY CONTEXT AT ATUEL RIVER VALLEY: CAÑADA SECA-1 ARCHAELOGICAL RECORD (SAN RAFAEL, MENDOZA)}

\section{ABSTRACT}

The goal of this paper is to describe an archaeological burial site with commingled skeletal remains, named Cañada Seca-1 (MNI=24), located in the Atuel River valley and dating from ca. 1500 years BP. This is performed by integrating well-known and recently developed lines of inquiry. Based on this record, we discuss interpretations related to cultigen presence and its implications in human subsistence and mobility, in an area traditionally characterized as the pre-Hispanic agricultural limit, ca. 2200 years BP. Results show that the individuals did not developed a farming strategy, although maize seems to have been part of their diet. In turn, the new information about mortuary behavior in the Atuel River valley provides new insights regarding mobility, as well as the use of utilitarian (lithics) and nonutilitarian (lithic and malacological ornaments) technology that went beyond these peoples' life into their death.

Keywords: prehispanic agriculture - diet - mobility - stable isotopes - Mendoza

\section{INTRODUCCIÓN}

El objetivo principal de este trabajo es presentar y evaluar por primera vez de manera articulada la información sobre el sitio Cañada Seca-1 (CS-1) fechado en ca. 1500 años AP: se trata de un área de entierro múltiple localizada en el valle del Atuel, que incluye un número mínimo de veinticuatro individuos acompañados de ornamentos, instrumentos líticos y restos de fauna. El análisis aquí propuesto integra numerosas líneas de evidencia-patrones de entierro, características de los restos óseos de las personas enterradas, dieta y movilidad a través de información isotópica, análisis de restos arqueofaunísticos y estudios de artefactos líticos y ornamentales enterrados en el sitio- que aportan información sobre sus tecnologías de producción e implicancias socioeconómicas en este contexto mortuorio.

El registro arqueológico del centro occidente argentino constituye un referente empírico para entender las características y causas del proceso de dispersión agrícola en América del Sur. Las investigaciones realizadas hasta el momento presentan a esta región como uno de los límites meridionales en el registro de cultígenos prehispánicos en la vertiente oriental andina de América (Lagiglia 1982; Gil 1997-98; Lema et al. 2012; Bernal et al. 2016a; Gil et al. 2018; Llano et al. 2019). Específicamente, el valle del río Atuel ha sido propuesto como el límite meridional hasta donde llegaron los primeros asentamientos agrícolas alrededor de 2200 años AP (Lagiglia 1982). Las ocupaciones de estos primeros agricultores fueron incluidas en la definición de la cultura Atuel II que se extendió temporalmente hasta ca. 1800 años AP (Lagiglia 1982). Tanto los documentos 
históricos como la interpretación del registro arqueológico señalan la presencia de grupos cazadores-recolectores para los momentos de contacto hispano-indígena, $c a$. 500 años AP (Lagiglia 1968; Gil y Neme 2010; Giardina et al. 2015). Los estudios arqueológicos relativos al proceso de agriculturización han destacado tres aspectos importantes. Por una parte, si bien en un primer momento (cultura Atuel II) se registra una abundancia de plantas domésticas (e.g., Gruta del Indio), su papel en la dieta humana habría sido poco relevante (Gil 2003; Peralta 2019). Por otra parte, en épocas históricas (cultura Atuel I) no hay mención de poblaciones agricultoras, por lo que la obtención de recursos domesticados se pudo haber dado por intercambio con vecinos productores de alimentos (Lagiglia 1968; Gil 1997-98). Finalmente, la secuencia cronológica-cultural clásica en este valle no definió unidades culturales que sucedieron entre Atuel II (2200 a 1800 años AP) y Atuel I (300 años AP), de modo que han quedado aproximadamente 1500 años sin una asignación del registro arqueológico a las categorías tradicionales de la región, provocando una suerte de "hiatus cultural". Aunque implícitamente se asumieron para este lapso ocupaciones de agricultores asociados a las culturas Agrelo y Viluco en el área del valle del Atuel (definidas originalmente para el registro arqueológico localizado en el norte de Mendoza; Lagiglia 1968, 2001), aún permanece la necesidad de profundizar en el registro arqueológico posterior a los 2000 años AP. Esto permitiría comprender la dinámica de ocupación humana en un contexto en el que estarían interactuando estrategias basadas en la caza-recolección y la producción de alimentos. El sitio Cañada Seca-1 tiene un marco cronológico que lo ubica entre las unidades culturales Atuel I y Atuel II, por lo que la discusión de su registro resulta significativa para entender los momentos posteriores al ingreso de los cultígenos en el área. En un trabajo previo, Gil y colaboradores (2018) exploraron de manera preliminar las tendencias isotópicas en este sitio y las integraron con la información disponible para otros dos sitios cercanos temporal y espacialmente, Jaime Prats-1 y Rincón del Atuel-1. En dicha oportunidad los datos se analizaron en escala más amplia, con menor detalle y sin considerar las particularidades contextuales inherentes al registro de Cañada Seca-1. En este trabajo, se presenta y describe el registro óseo humano recuperado en este sitio y se profundiza en aspectos relativos a la dieta y al patrón de movilidad de los grupos que lo ocuparon, a través del análisis de isótopos estables. Concomitantemente, se ofrece información inédita relativa a las condiciones del hallazgo, características del registro esqueletal humano, ornamentos, instrumentos líticos y registro óseo no humano.

\section{DIETA Y MOVILIDAD HUMANA EN LA FRONTERA AGRÍCOLA: IDEAS, MODELOS Y REGISTRO EN EL CENTRO OCCIDENTE ARGENTINO}

En el sector norte del centro occidente de Argentina los estudios arqueológicos de las décadas de 1970 y 1980, propusieron la incorporación de plantas domésticas hacia ca. 4000 años AP (Lagiglia 1968; Gambier 1980; Bárcena et al. 1985). Se definieron, clásicamente, distintas unidades culturales que sustentaron un modelo general de agriculturización apoyado en el registro arqueológico de tres sectores: suroeste de San Juan (Gambier 1977), noroeste de Mendoza (Bárcena et al. 1985; Bárcena 2001) y valle medio del Atuel (Lagiglia 1982, 2001). En estos sectores, la cronología y las características del proceso habrían sido semejantes, variando principalmente la denominación regional que los investigadores dieron a cada contexto. En una primera etapa ( $c a$. 4000 años AP), algunas veces vinculada con cazadores-recolectores del Arcaico tardío, el registro señala escasos restos de cultígenos, cuya cronología se les asignó por asociación contextual con otros materiales fechados (Lagiglia 1968, 1982, 1999; Bárcena et al. 1985). Se señala una rápida dispersión geográfica de plantas domésticas en esta etapa inicial, aunque mantuvieron un papel poco significativo en la dieta humana. Para la segunda etapa, entre $c a$. 2300 y 1700 años AP, se incrementa la presencia de restos de vegetales domesticados, aunque los grupos humanos no 
habrían abandonado la explotación de recursos silvestres (Gambier 1977; Lagiglia 1982, 2001; Bárcena et al. 1985; Bárcena 2001). En algunas áreas los cambios de esta etapa involucraron un crecimiento demográfico significativo y nuevas organizaciones socioculturales que se cristalizaron principalmente en grupos con menor movilidad residencial, incluso sedentarios (Gambier 1977; Lagiglia 1982, 2001; Bárcena et al. 1985, 2001). Estos cambios se asociaron con innovaciones posteriores en la historia regional, como el desarrollo de los primeros sistemas de irrigación en la zona de San Juan (Gambier 1977). La temporalidad de los cambios habría significado una transición lenta a una mayor dependencia de la agricultura (en algunos casos atada al pastoreo).

El valle del Atuel fue propuesto como el límite de estos productores, que convivieron hasta épocas históricas con cazadores-recolectores asentados hacia el sur (Gil y Neme 2010). Esta situación de coexistencia entre sistemas de subsistencia aparentemente contrastantes en el valle del Atuel podría explicar la falta de patrones claros en el registro como los definidos en las otras dos áreas mencionadas. En esta área, la continuidad de los estudios y la incorporación de otros enfoques teóricos han ajustado algunos aspectos de estos modelos clásicos (Neme y Gil 2012). Primero, la presencia de plantas domésticas no habría significado el establecimiento de la agricultura propiamente dicha. Hay una amplia gama de estrategias que deberían ser consideradas y que van desde el intercambio con vecinos productores, pasando por productores de baja escala, hasta el desarrollo de una fuerte agricultura local (Gil et al. 2020). Segundo, las plantas domésticas no siempre tuvieron la misma función, incluso en algunos momentos pudieron ser materializaciones de aspectos simbólicos sin implicancias para la dieta humana (Gil 1997-98, 2003). Tercero, el registro espacial y temporal muestra un mosaico de estrategias que incluyeron la producción de alimentos en distinto grado, o la excluyeron a pesar de la disponibilidad (Gil et al. 2018). Gil y colaboradores (2018) mostraron cómo la importancia del maíz en la dieta humana varió entre poblaciones próximas espacial y temporalmente, incluyendo en esta variación una reversión a estados previos. Los modelos derivados de la ecología evolutiva señalan que la incorporación de plantas domésticas se relaciona con una caída en la frecuencia de recursos silvestres (Winterhalder y Golad 1997; Barlow 2002; Corbat et al. 2020) ya sea por sobre explotación o cambio climático. Hay varios escenarios que pueden explicar la forma y la posterior evolución de dicha incorporación teniendo en cuenta la estructura de los recursos, las variaciones climáticas y la dinámica poblacional humana (Winterhalder y Goland 1997; Winterhalder y Kennett 2006, 2020). Esta perspectiva teórica ha influido en la manera en que las investigaciones llevadas a cabo en el valle del Atuel buscan entender las causas y el contexto en que los cultivos se incorporaron a la dieta humana, así como las consecuencias posteriores en el sistema humano-ambiental. Cañada Seca-1 se emplaza en este valle y su estudio intenta ofrecer información que permita ajustar el entendimiento de este proceso evolutivo.

\section{CS-1: LOCALIZACIÓN, ANTECEDENTES, CONTEXTO Y PATRONES DE ENTIERRO}

El sitio Cañada Seca 1 (en adelante CS-1) se localiza en el valle medio del río Atuel (S3445"2,6" y O69¹'18,3"; 596 m s.n.m.) al sur de la provincia de Mendoza (figura 1). Se trató de un hallazgo fortuito ocurrido en el año 1991, como consecuencia del movimiento de suelos para extracción de arcilla destinada a la fabricación de ladrillos. En ese mismo año, el equipo del Museo de Historia Natural de San Rafael (MHNSR) desarrolló los trabajos de rescate y la documentación de campo del sitio. CS-1 habría sido perturbado previamente por los lugareños en la búsqueda de "objetos de valor económico" (Lagiglia 1991). Las condiciones de hallazgo y la ausencia de información acerca de las modalidades de entierro condujeron a que el sitio fuera definido como un entierro múltiple mezclado, compuesto por elementos óseos disociados (Peralta 2015) (figura 2). Cabe aclarar que no se cuenta con anotaciones o registro escrito de campo, ni 
se pudo confirmar la ubicación exacta del área de entierro, lo que impidió conocer en detalle el contexto estratigráfico y el proceso de formación del sitio o las asociaciones en el interior del área de entierro. Junto con los restos humanos se hallaron restos de fauna, objetos de ornamentación personal (cuentas de collar y tembetás) y puntas de proyectil. Aunque fueron recuperados en el mismo contexto, se desconoce la asociación precisa entre estos elementos y los esqueletos.

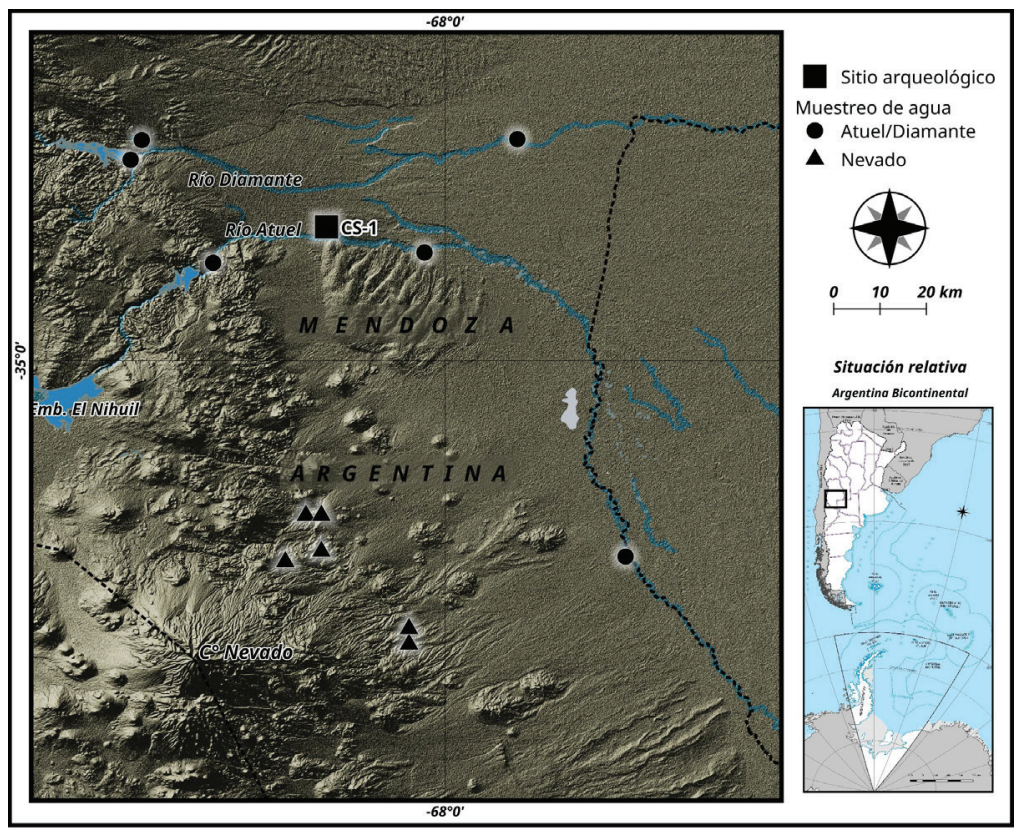

Figura 1. Ubicación del sitio Cañada Seca-1 (CS-1; valle medio del Atuel, San Rafael-Mendoza)

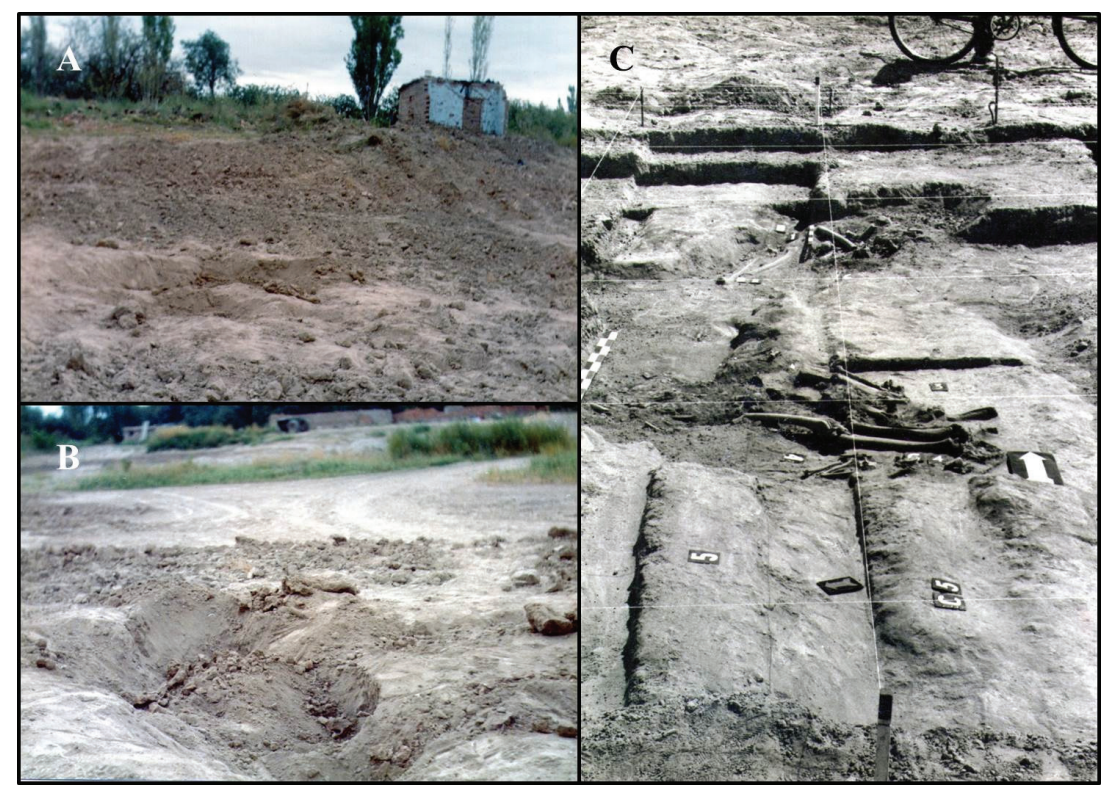

Figura 2. Vista general de las excavaciones arqueológicas en Cañada Seca. (Foto Archivo MHNSR) 
La cronología del sitio se definió sobre la base de cinco fechas radiocarbónicas, todas obtenidas sobre hueso humano (tabla 1). La figura 3 muestra detalles de la calibración de cada fechado, basados en la curva SHcal20 (Hogg et al. 2020). Tres de ellos se realizaron a través del método AMS en la Arizona Radiocarbon Facility, mientras que los dos fechados restantes fueron obtenidos mediante la técnica de espectrometría de centelleo líquido, en el Laboratorio de Tritio y Radiocarbono (Universidad Nacional de La Plata, Argentina). Las fechas radiocarbónicas varían entre 1420 y 1790 años ${ }^{14} \mathrm{C}$ AP, mientras que la mediana calibrada oscila entre 1286 y 1656 años cal. AP, con rangos máximos de 1180 y 1706 años cal. AP, si se considera un sigma. Se identificaron diferencias estadísticamente significativas al evaluar todo el conjunto de fechados, cuya media es de $1588 \pm 20$ años ${ }^{14} \mathrm{C}$ AP $\left(X^{2}=9,49 ; 4\right.$ grados de libertad $)$. Si no se consideran la fecha más antigua y la más reciente, procesadas por espectrometría de centelleo, la media calculada a partir de las restantes, obtenidas por AMS, es similar $\left(1565 \pm 25\right.$ años $\left.{ }^{14} \mathrm{C} A P\right)$, pero en este caso no se observan diferencias significativas entre las tres fechas $\left(X^{2}=5,99 ; 2\right.$ grados de libertad) y su calibración ofrece una mediana de 1398 años cal. AP, con un rango de un sigma entre 1314 y 1514 años cal. AP (tabla 1; figura 3). Dada la diferencia de metodología, de procedimiento en la obtención de colágeno y la falta de corrección por fraccionamiento isotópico esta última interpretación es más sólida que la que incluye la totalidad de las muestras.

Tabla 1. Fechados radiocarbónicos obtenidos en CS-1 (todos sobre restos humanos)

\begin{tabular}{|c|c|c|c|c|}
\hline Código Laboratorio & Años ${ }^{\mathbf{1 4}} \mathbf{C}$ AP & DS & $\begin{array}{c}\text { Mediana Calibrada años } \\
\text { AP }\end{array}$ & Referencias \\
\hline LP-1184 & 1790 & 50 & $1588(1656) 1706$ & Gil y Neme (2010) \\
\hline AA-93795 & 1592 & 45 & $1374(1442) 1514$ & Gil et al. $(2014)$ \\
\hline AA-93793 & 1566 & 45 & $1321(1409) 1475$ & Gil et al. $(2014)$ \\
\hline AA-93794 & 1538 & 45 & $1314(1379) 1413$ & Gil et al. $(2014)$ \\
\hline LP-1374 & 1420 & 60 & $1180(1286) 1349$ & Gil y Neme $(2010)$ \\
\hline
\end{tabular}

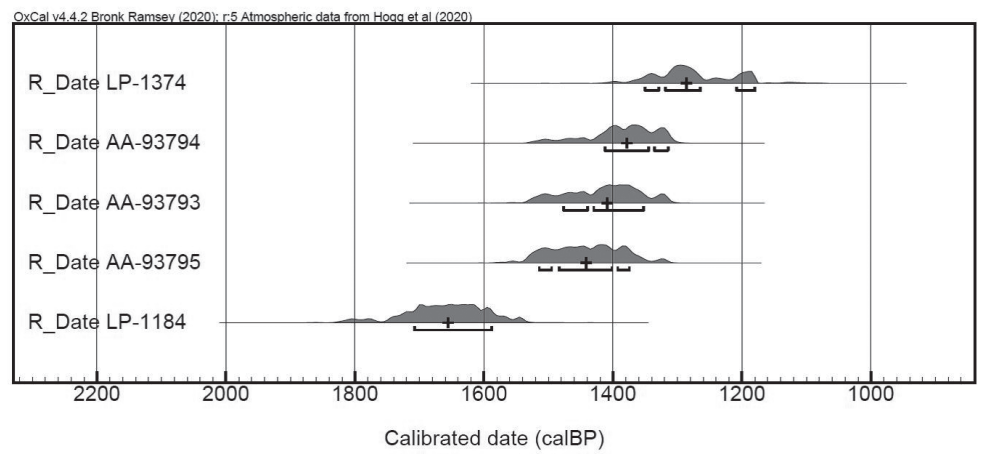

Figura 3. Fechados calibrados de CS-1

Hasta el momento, el avance de las investigaciones bioarqueológicas en el sitio se abocó al análisis de indicadores no específicos de dieta y salud (desgaste dental, caries e hipoplasia), morfometría craneofacial y dental, paleodemografía y marcadores de uso del cuerpo (Novellino y Guichón 1997-98; Novellino et al. 2004; Novellino y Gil 2007; Gil et al. 2018; Peralta 2020). En CS-1 no se identificaron hipoplasias del esmalte dental ni caries, mientras que sí se registró una alta prevalencia de desgaste (avanzado para más del 50\% de los individuos), lo que resulta 
compatible con lo observado en grupos con subsistencias basadas en la caza-recolección (Cohen y Armelagos 1984; Novellino y Guichón 1997-98; Novellino et al. 2004; Novellino y Gil 2007). En el mismo sentido, estudios de morfometría craneofacial y dental mostraron afinidades con poblaciones cuya subsistencia implicó principalmente la explotación de recursos silvestres (Gil et al. 2018). En cuanto al uso del cuerpo, estudios recientes mostraron que las prevalencias de lesiones articulares y cambios entésicos, registradas en miembros inferiores, permiten suponer niveles altos de exigencia física vinculados con la movilidad (lesiones articulares $=37,5 \%$; cambios entésicos $=44 \%$ ), aunque menores a lo observado en poblaciones de cazadores-recolectores típicos (lesiones articulares $=44,9 \%$; cambios entésicos=87,9\%) (Peralta 2015, 2017, 2020). Por otra parte, estudios paleodemográficos preliminares refuerzan la interpretación de una ocupación de cazadores-recolectores en CS-1 en la que los recursos domésticos no habrían impactado de manera determinante en el estilo de vida (Peralta 2019). La ausencia de individuos menores a 1 año de edad y el Índice de Juventud ${ }_{15} \mathrm{P}_{5}(3 / 21=0,14)$, obtenidos a partir de las distribuciones de edad de muerte, indican la ausencia de crecimiento demográfico esperable en un contexto de transición hacia la agricultura (Binford y Chasko 1976; Buikstra et al. 1986; Bocquet-Appel 2002; Bocquet-Appel y Dubouloz 2003, 2004).

Los resultados mencionados se obtuvieron en el marco de investigaciones de escala regional con poca resolución a nivel de sitio. En este trabajo se articulan, integran y reinterpretan estas tendencias explorando a nivel individual y grupal los datos de isótopos estables con mayor exhaustividad, profundizando en el rol del maíz en CS-1. De esta manera se espera aportar al entendimiento de la dinámica de la incorporación de cultígenos en el área.

\section{CONSIDERACIONES METODOLÓGICAS}

\section{Tamaño y composición de la muestra ósea humana}

En primer lugar, se caracterizó cuantitativamente la muestra ósea humana, lo que permite visibilizar posibles sesgos culturales y/o tafonómicos en relación con la representación diferencial de los elementos. Para estimar cuán fragmentado está cada elemento y cuán completo está el esqueleto se calcularon dos índices: el de Fragmentación (IF) y el de Completitud (IC). Asimismo, se obtuvieron valores correspondientes a las medidas de abundancia taxonómica y relativa: número mínimo de especímenes (NISP), número mínimo de elementos (NME) y unidades anatómicas mínimas, absolutas y porcentuales (MAU y MAU\%). El NISP se obtuvo a través de la contabilización de todos los especímenes, incluyendo elementos fragmentados y fragmentos identificables y lateralizables. Para el NME se consideraron las frecuencias de cada tipo de hueso, según su lateralidad, segmento representado (e.g., epífisis proximal, distal, diáfisis, tercio medio de la diáfisis, tercio proximal de la diáfisis) o parte diagnóstica identificada (e.g., ilion, rama isquiopúbica; cuerpo, arco dorsal). Los valores de MAU se obtuvieron dividiendo el NME de cada unidad anatómica (i.e., cráneo, húmero, vértebras cervicales) por su frecuencia en el esqueleto completo, mientras que los valores de MAU\% se estimaron proporcionalmente considerando el valor de MAU más alto (Mengoni Goñalons 1988, 1999; Lyman 1994; Mondini 2003). EL IF se obtuvo a partir de la razón entre el NME y el NISP, obteniendo resultados en una escala de 0 a 1: cuanto mayor sea el valor obtenido, menor es la fragmentación (Mondini 2003; Luna 2008). De manera complementaria a estas mediciones, se llevó a cabo un relevamiento tafonómico exploratorio en el que se consideraron las siguientes variables: marcas de raíces, roedores y carnívoros, grado de meteorización, precipitaciones químicas (óxido de manganeso y carbonato de calcio), fracturas y pérdida ósea, y marcas de origen antrópico moderno y no moderno. Se consideró la presencia/ ausencia de las variables mencionadas a nivel del elemento anatómico, aunque contemplando 
a nivel general (no sistemático) las distribuciones e intensidad de impacto de algunos agentes (i.e., raíces, precipitaciones químicas). Se siguieron las recomendaciones presentes en Buikstra y Ubelaker (1994), White y Folkens (2005) y González (2014).

Junto con las medidas de abundancia se analizaron las características de la muestra en términos de sexo y edad, y se estimó el número mínimo de individuos (NMI). La estimación del sexo de los adultos se llevó a cabo a partir del relevamiento del coxal y del cráneo, siguiendo los criterios recopilados en Buikstra y Mielke (1985) y Buikstra y Ubelaker (1994), y los propuestos por Bruzek (2002). Asimismo, se analizaron variables métricas de huesos largos y del calcáneo (Steele 1976; Berrizbeitia 1989; Holland 1991; Bruzek 1995; Silva 1995; Introna et al. 1997; Trancho et al. 1997, 2000; Seidemann et al. 1998; Wilbur 1998; Alemán Aguilera et al. 2000; López-Bueis et al. 2000; Robledo et al. 2000; Murphy 2002; Ríos Frutos 2003; Cowal y Pastor 2008). Los datos obtenidos fueron procesados posteriormente considerando la variabilidad dimórfica de los individuos hacia el interior de la muestra, vinculando los valores más bajos con los individuos femeninos y los más altos con los masculinos (Peralta 2019). En esta instancia también se consideraron valores de referencia, obtenidos en muestras arqueológicas de la región en las que el sexo se estimó a través de variables morfológicas del coxal.

La edad de muerte en los adultos se estimó a partir del registro de los cambios producidos en la sínfisis púbica y la superficie auricular de los coxales, siguiendo los criterios propuestos por Todd (1921 a y b), Lovejoy et al. (1985), Meindl y Lovejoy (1989) y Meindl et al. (1980). De manera complementaria se aplicó el método basado en la obliteración de las suturas craneanas para el sistema latero-anterior (Meindl y Lovejoy 1985). La estimación de la edad en subadultos se realizó considerando la longitud de huesos largos y los estadios de fusión de epífisis y apófisis, a partir del compendio realizado por Scheuer y Black (2000).

\section{Análisis de los ornamentos corporales}

Las cuentas y tembetás hallados junto a los esqueletos se observaron en su totalidad a ojo desnudo y con lupa de mano; y, en casos específicos, con estereomicroscopio (Nikon SMZ con aumentos entre 50X y 200X). Se registraron sistemáticamente las siguientes variables: forma, tamaño, materia prima y estado de conservación. Debido a la gran cantidad de cuentas halladas y a su registro en "grupos"1, se realizó un estudio preliminar de su tamaño midiendo en cada grupo el diámetro de la pieza más grande (diámetro máximo) y el diámetro de la pieza más pequeña (diámetro mínimo). Para obtener un tamaño promedio estimativo de cada grupo, se extendió el total de piezas de cada grupo para observarlas simultáneamente, eligiendo aquella que registrara el diámetro más frecuente, que se tomó como representación del tamaño promedio del conjunto (diámetro más frecuente). El estado de conservación entero/fragmentado fue observado a escala del grupo, evaluando visualmente si la mayoría de las piezas estaban enteras, muy fragmentadas o si solo unas pocas piezas estaban fragmentadas. Las materias primas fueron identificadas mediante la observación de las piezas a ojo desnudo y con magnificación de diversos aumentos, y se compararon con muestras naturales previamente conocidas para la región y zonas adyacentes.

\section{Análisis lítico: puntas de proyectil}

El análisis del conjunto lítico de CS-1 (n=8) se realizó utilizando el enfoque tecno-tipológico, sensu Aschero (1975, 1983), y se consideraron las modificaciones realizadas por Aschero y Hocsman (2004). En cada pieza se identificaron diversos atributos: tipo de materia prima, según determinación por petrografía macroscópica, y su calidad para la talla (sensu Aragón y Franco 
1997); reserva de corteza (presente o ausente); estado de fragmentación (entero o fracturado); tamaño (largo, ancho y espesor, en milímetros) según eje morfológico; y peso de cada pieza en gramos, mediante el uso de balanza digital (con error de $0,1 \mathrm{gr}$ ). Debido a que el grupo tipológico de todas las piezas es el de las puntas de proyectil, en ellas se analizaron las siguientes variables: técnica de manufactura, continuidad y profundidad de los lascados, forma perimetral del limbo, forma secundaria del borde del limbo, serie técnica (definida por el ancho de la boca de los lascados y por la extensión sobre las caras de la pieza), sección transversal (en vista sagital), reactivación (presente o ausente) y tratamiento del ápice (sensu Aschero 1975, 1983). En las piezas cuyo subgrupo tipológico identificado fue el de las puntas de proyectil apedunculadas, se evaluaron otros atributos morfológicos como la forma de la base del limbo y el tratamiento técnico diferencial de la base.

\section{Análisis zooarqueológico}

El conjunto arqueofaunístico fue analizado considerando la composición y estructura taxonómica y anatómica, así como las características tafonómicas (Lyman 1994). Para realizar estas determinaciones se utilizó material óseo comparativo disponible en la colección del Laboratorio de Ecología Humana del Instituto de Evolución, Ecología Histórica y Ambiente (IDEVEA) -San Rafael, Mendoza- y del Centro Regional de Investigaciones Científicas y Transferencia Tecnológica de La Rioja (CRILAR) -Anillaco, La Rioja-, así como literatura específica (Prevosti et al. 2009, 2013; Fernández et al. 2011). Las asignaciones taxonómicas correspondientes a cánidos también fueron corroboradas por el doctor F. J. Prevosti. Se presentan solo los valores de NISP, dado que representan muy bien las características de la muestra, evitando los errores generados en el manejo de datos más complejos como el NMI (Lyman 1994). Para las variables tafonómicas pre y postdepositacionales se aplicó la metodología desarrollada en trabajos previos (Behrensmeyer 1978; Binford 1981; Todd y Rapson 1988; Andrews 1990; Fernández et al. 2017).

\section{Análisis de isótopos estables}

Se presentan los valores de $\delta^{13} \mathrm{C}$ y $\delta^{15} \mathrm{~N}$ basados en el procesamiento de la fracción orgánica (colágeno) y de $\delta^{13} \mathrm{C}$ obtenidos de la fracción inorgánica (hidroxiapatita o bioapatita). Los valores de $\delta^{13} \mathrm{C}$ y $\delta^{18} \mathrm{O}$ se calcularon utilizando el estándar V-PDB, mientras que el de $\delta^{15} \mathrm{~N}$ se calculó con el estándar AIR (Samec et al. 2019). El colágeno y la bioapatita se obtuvieron procesando las muestras en el Laboratorio de Paleoecología Humana del IDEVEA, siguiendo el protocolo descrito en Coltrain y Leavitt (2002) y Gil et al. (2011). Las mediciones se efectuaron en SIRFER de la University of Utah (código MSR) y en la University of South Florida (código USF). Las mediciones en SIRFER se determinaron utilizando un espectrómetro Finnigan Delta Plus acoplado mediante Conflo III a un analizador elemental Carlo Erba Model 1110. Como estándar para la normalización de las mediciones se emplearon los patrones USGS40 y USGS41; la precisión analítica es de $0,1 \%$ o para las relaciones isotópicas de carbón (V-PDB) y de $0,2 \%$ o para nitrógeno (AIR). Para las mediciones en la University of South Florida se utilizó un analizador elemental CNH acoplado a Finnigan MAT. La precisión analítica de la espectrometría de masas para la razón isotópica es $\pm 0,1 \%$ para $\delta^{13} \mathrm{C}$ y $\pm 0,2 \%$ para $\delta^{15} \mathrm{~N}$. Para medir carbonatos se empleó el equipo acoplando un Kiel III. Se obtuvieron también valores de $\delta^{13} \mathrm{C}$ y $\delta^{15} \mathrm{~N}$ de colágeno de una muestra ósea de cánido. Las mediciones sobre esta última se realizaron en el Laboratorio de Isótopos Estables en Ciencias Ambientales (LIECA, San Rafael, Mendoza) a través del espectrómetro de masas de flujo continuo Thermo Scientific DELTA V acoplado vía ConFlo IV al Analizador Elemental 
Flash 2000. Para este equipo la precisión analítica es de 0,1\% para las relaciones isotópicas de carbón (V-PDB) y de 0,2\%o para nitrógeno (AIR).

Para evaluar la integridad de las muestras de colágeno se analizó en cada una de ellas la relación C:N siguiendo los criterios de Ambrose (1990) y se aceptaron aquellas cuyos valores estén comprendidos entre 2,9 y 3,6. Por otra parte, se ha demostrado que existen procesos diagenéticos de recristalización que pueden afectar las relaciones isotópicas de los carbonatos y fosfatos que componen la fracción inorgánica del hueso (Hedges et al. 2005). Dado que para la bioapatita no se han desarrollado protocolos semejantes a los del colágeno para controlar la calidad del dato isotópico, se tuvieron en cuenta los valores de la relación C:N y la coherencia interna de los valores obtenidos como parámetro de la confiabilidad de los resultados en la fracción inorgánica.

De acuerdo con información arqueológica de base de investigaciones previas (Giardina et al. 2015; Gil et al. 2018, 2020; Otaola et al. 2018), los recursos considerados en las reconstrucciones dietarias fueron: guanaco (Lama guanicoe), armadillo (Zaedyus pichiy), maíz (Zea mays) como ejemplar $\mathrm{C}_{4}$, algarrobo (Prosopis sp.) como ejemplar $\mathrm{C}_{3}$, pescado y huevo de reído. Para conocer los valores de isótopos estables de estos recursos consultar la tabla suplementaria 2 (http://doi. org/10.5281/zenodo.4769808). En los gráficos bivariados se corrigió el fraccionamiento de dieta a tejido óseo humano utilizando los siguientes factores de discriminación: para animales, $\delta^{13} \mathrm{C}_{\mathrm{col}}$ $+1,0 \pm 0,3 / \delta^{15} \mathrm{~N}+4,6 \pm 1,0$ (Bocherens y Druker 2003; Bernal et al. 2016 b; Cheung y Szpak 2020; Gil et al. 2020); para plantas, $\delta^{13} \mathrm{C}_{\mathrm{col}}+3,1 \pm 1,4 / \delta^{15} \mathrm{~N}+4 \pm 1$ (Bernal et al. $2016 \mathrm{~b}$; Gil et al. 2020); para animales, $\delta^{13} \mathrm{C}_{\text {apa }}+8,0 \pm 0,1 / \delta^{15} \mathrm{~N}+4,0 \pm 0,1$ (Tykot et al. 2009; Bernal et al. 2016b; Gil et al. 2020); para plantas, $\delta^{13} \mathrm{C}_{\text {apa }}+11,1 \pm 0,1 / \delta^{15} \mathrm{~N}+4,6 \pm 0,1$ (Tykot et al. 2009; Bernal et al. 2016 b; Gil et al. 2020). Esta elección se realizó considerando que son los fraccionamientos que se han empleado en las últimas investigaciones regionales, lo que permite futuras comparaciones. Además, estos valores contemplan la corrección de músculo a colágeno de tejido óseo de los animales.

Para la interpretación de los datos isotópicos se utilizaron también modelos bayesianos de mezcla de dieta. Estos modelos permiten ajustar la interpretación de las dietas profundizando el análisis bivariado mediante modelos de combinación estadística donde se explora la probabilidad de los aportes de distintos recursos (Moore y Semmens 2008; Phillips 2012; Parnell et al. 2013; Giardina et al. 2014; Phillips et al. 2014; Bernal et al. 2016; Gil et al. 2018). Para ello se utilizó el software Food Reconstruction Using Isotopic Transferred Signals (FRUITS) desarrollado por Fernandes et al. (2014). Para mantener la coherencia con lo que este autor propone para la aplicación de su modelo de mezcla, se utilizaron los siguientes factores de discriminación: 5,5\%o para $\delta^{15} \mathrm{~N}$, de $4,8 \%$ o para $\delta^{13} \mathrm{C}_{\text {col }}$ y de $10,1 \%$ o para $\delta^{13} \mathrm{C}_{\text {apa }}$ (Fernandes 2016). Aparte de los factores de discriminación de dieta a tejido óseo humano, los valores de $\delta^{13} \mathrm{C}$ y $\delta^{15} \mathrm{~N}$ obtenidos sobre colágeno óseo de animales fueron corregidos de acuerdo con Fernandes (2016) del siguiente modo: para herbívoros, $\Delta^{13} \mathrm{C}_{\text {proteína-colágeno }}=-2 \%$; $\Delta^{15} \mathrm{~N}_{\text {proteina-colágeno }}=+2 \% ; \Delta^{13} \mathrm{C}_{\text {colágeno-lípidos }}=-8 \%$; para peces, $\Delta^{13} \mathrm{C}_{\text {proténa-colágeno }}=-1 \% \circ ; \Delta^{15} \mathrm{~N}_{\text {proteina-colágeno }}=+2 \% \circ ; \Delta^{13} \mathrm{C}_{\text {colágeno-lípidos }}=-7 \%$. Los valores de las plantas fueron corregidos considerando los siguientes factores: $\Delta^{13} \mathrm{C}_{\text {vegetal total-proteínas }}=-2 \%$; $\Delta{ }^{13} \mathrm{C}_{\text {vegetal total-carbohidratos }}=+0,5 \%$ (Fernandes et al. 2015). Asimismo, el modelo considera las concentraciones relativas de los diferentes recursos en términos de proteínas y energía, presentes en los músculos de los animales. Se calculan a partir de tablas nutricionales utilizando las fórmulas indicadas a continuación, de acuerdo con la propuesta de Nakamura et al. (1982) y Morrison et al. (2000) (Fernandes et al. 2015; Kochi 2017). Bajo la asunción de que el carbono compone el $52,4 \%$ de las proteínas, el 44,4\% de los carbohidratos y el 76,8\% de los lípidos, las fórmulas por utilizar son las siguientes (Nakamura et al. 1982; Morrison et al. 2000):

$\%$ proteína $=(0,524 x$ Proteínas $) /(0,524 x$ Proteínas $+0,444 \times$ Carbohidratos $+0,768 x$ Lípidos $)$

$\%$ energía $=(0,444 \times$ Carbohidratos $+0,768 x$ Lípidos $) /(0,524 x$ Proteínas $+0,444 \times$ Carbohidratos + 0,768xLípidos) 
Las proteínas, carbohidratos y lípidos corresponden a los gramos presentes de cada uno en 100 gr del recurso considerado. Esta información se obtuvo de United States Department of Agriculture (2015), Urbistondo y Pereyra (2015) y Boeri et al. (2017). Las estimaciones generadas a partir de FRUITS se realizaron en términos de la contribución calórica de cada recurso -Food (\%)- de acuerdo con Fernandes et al. (2015). Para hacer funcionar el modelo se realizaron combinaciones de cuatro a cinco de estos recursos, variando la fuente proteica y manteniendo la fuente vegetal $\mathrm{C}_{3}$ y $_{4}$. Para conocer los valores de isótopos estables de los recursos en el programa y sus concentraciones en términos de proteína y energía consultar la tabla suplementaria 2. La elección de trabajar con este software se realizó considerando que fue diseñado para modelizar la dieta a partir de casos arqueológicos y que permite considerar las proporciones de los macronutrientes (proteínas, carbohidratos y lípidos) en las que contribuye cada recurso a la dieta (Fernandes et al. 2014, 2015). En este sentido, provee reconstrucciones más robustas en grupos con dietas más o menos especializadas, como aquellas que son ricas en carbohidratos o lípidos (Cheung y Szpak 2020). Es esperable, entonces, el uso de este modelo en contextos en los que se quiera estimar la importancia del maíz en la dieta, por ejemplo. Por otra parte, en un trabajo previo (Gil y colaboradores) se intentaron modelizaciones a través del modelo MixSIAR, por lo que realizar nuevas exploraciones empleando otro modelo de mezcla permitirá comparar los alcances de cada propuesta y su impacto en las reconstrucciones de dieta.

Los gráficos bivariados también se realizaron empleando los fraccionamientos sugeridos por Fernandes (2016) para evaluar similitudes y diferencias en las tendencias obtenidas con ambos grupos de fraccionamientos.

Para evaluar las tendencias en la movilidad se convirtieron los valores de $\delta^{18} \mathrm{O}$ de carbonatos humanos a valores de aguas usando las relaciones entre los estándares VSMOW y VPDB, los valores de $\delta^{18} \mathrm{O}$ de carbonatos (c) y fosfatos (p) (VSMOW), y los valores de fosfatos y aguas bebidas (ab) (VSMOW), como se muestra en las siguientes ecuaciones (siguiendo los trabajos de Luz et al. 1984; Iacumin et al. 1996; Sharp 2007; Buzon et al. 2011; Ugan et al. 2012; Bernal et al. 2020):

$$
\begin{aligned}
& \delta^{18} \mathrm{O}_{\mathrm{c}} \text {, vSMOW }=\left(1,03092 * \delta^{18} \mathrm{O}_{\mathrm{c}, \mathrm{VPDB}}\right)+30,92 \% \text { o } \\
& \delta^{18} \mathrm{O}_{c} \text {, vSMOw }=\left(8,50 \% \text { o }+\delta^{18} \mathrm{O}_{\mathrm{p}, \text { vSMOW }}\right) / 0,98 \\
& \delta^{18} \mathrm{O}_{\mathrm{ab}}=\left(\delta^{18} \mathrm{O}_{\mathrm{p}, \text { vSMOW }}-22,70 \%\right) / 0,78
\end{aligned}
$$

Los valores de aguas provienen de muestreos realizados en tres grupos de fuentes (río Atuel, río Diamante y Cerro Nevado), cuyos resultados se publicaron en trabajos previos (Gil et al. 2012, Ugan et al. 2012).

\section{RESULTADOS}

\section{Tamaño y composición de la muestra ósea humana}

Sobre un total de 3.470 especímenes óseos humanos recuperados en los trabajos de campo, se obtuvo un NME de 1.096, con representación de todos los elementos anatómicos del esqueleto humano, aunque en distintas frecuencias (tabla suplementaria 1: http://doi.org/10.5281/ zenodo.4769808). En cuanto a la representatividad de las unidades anatómicas, las que exhibieron mayores porcentajes de supervivencia (entre 66 y 100\%) fueron el húmero, el cúbito, el fémur y la tibia (figura 4). El cráneo, la cintura pélvica, la cintura escapular, la columna vertebral, el radio, la rótula, el peroné, los metacarpos, los metatarsos y los tarsos presentaron porcentajes de supervivencia de $65-33 \%$. En las restantes unidades se observaron los porcentajes de supervivencia más bajos $(32-0,1 \%)$. 

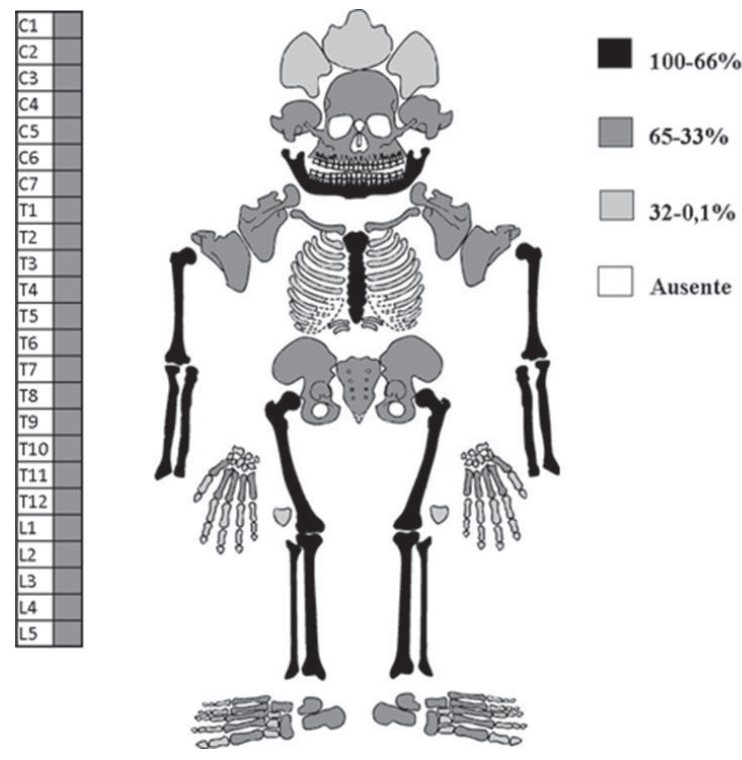

Figura 4. MAU\% correspondiente a Cañada Seca graficando la representatividad de los diferentes elementos anatómicos

El IF a nivel de cada elemento indica que el cráneo, las costillas y el coxal fueron los elementos más fragmentados. Resulta significativa la baja frecuencia de cráneos completos que componen la muestra, en relación con las mandíbulas y el resto de los elementos presentes. Considerando el potencial de preservación del cráneo, y que no están ausentes otros elementos de mayor fragilidad, esta diferencia de frecuencias y la alta fragmentación pueden asociarse con actividades de profanación por parte de los lugareños.

El relevamiento de variables tafonómicas indica que las raíces fueron los agentes que más afectaron a los restos. El 98,8\% presenta marcas atribuibles a raíces, desde coloración superficial hasta deterioro químico y corrosión. Dichas marcas se distribuyen de manera uniforme y extensiva en los elementos óseos en los que se presentan, aunque con mayor o menor intensidad de alteración química-mecánica. En muy bajas frecuencias se registraron marcas atribuibles a roedores y carnívoros $(0,03$ y $0,09 \%$, respectivamente). La pérdida ósea $(89,7 \%)$ y las fracturas postdepositacionales $(88,5 \%)$ se encuentran entre las variables más representadas. Considerando la alta prevalencia de marcas de raíces, es probable que una parte significativa de las fracturas y pérdidas óseas se expliquen como resultado de la acción de dicho agente.

Las señales vinculadas con el proceso de meteorización se atribuyeron al grado 1 en la escala de Behrensmeyer (1978) con una prevalencia del 0,94\%. No se registraron grados más severos de este fenómeno, lo que nos permitiría inferir que los episodios de eventual desentierro y exposición de los restos óseos no tuvieron lugar o fueron muy poco frecuentes y de corta duración. En cuanto a las precipitaciones químicas, la presencia de óxido de manganeso $(56,8 \%)$ se dio con mucha mayor frecuencia que la de carbonato de calcio $(9,8 \%)$. La distribución y cantidad del óxido de manganeso fue muy variable entre los distintos elementos óseos, yendo desde una distribución homogénea a una irregular o en parches, y desde pequeñas manchas dispersas a extensos "manchones" de coloración oscura. En el caso del carbonato de calcio el patrón de distribución fue mucho más acotado, presentándose en forma de pequeños parches de coloración blanquecina en algunos elementos. Las marcas de origen antrópico de carácter no moderno se registraron con un porcentaje del $0,4 \%$. Las huellas de actividad moderna fueron más fácilmente identificables y 
más numerosas $(3,1 \%)$. En estos casos las características de los cortes (pulimentados) y fracturas del hueso seco permitieron vincular las marcas con impactos accidentales durante la excavación y/o con el manejo posterior de los restos (e.g., extracción de material para análisis isotópicos).

La estimación del sexo y de la edad mediante el registro de variables morfológicas de la pelvis solo fue posible en catorce elementos. Se identificaron: un individuo femenino adulto joven, tres individuos masculinos y dos individuos femeninos adultos medios, y dos individuos masculinos adultos maduros. Se registraron solo tres cráneos, correspondientes a un masculino adulto medio y dos masculinos adultos maduros. En dos individuos pudo observarse la variación morfológica de la bóveda y se pudieron describir modificaciones culturales del cráneo. Mientras que el individuo AF-2019 presenta un aplanamiento plano-lámbdico, el individuo AF-2020 presenta un aplanamiento occipital (cf. Gil et al. 2018). El fémur resultó el elemento más frecuente arrojando un NMI de 18 individuos adultos (>20 años), 2 juveniles (15-19,9 años) y 1 niño (5-9,9 años). La tibia permitió además identificar 3 individuos infantiles (1-4,9 años). A partir de estos elementos del poscráneo se obtuvo un NMI de veinticuatro individuos, entre adultos, juveniles y subadultos.

\section{Análisis de ornamentos corporales}

La colección consta de 1.243 cuentas distribuidas en 20 grupos (grupo \#1 al grupo \#20) y 16 tembetás (que conforman el grupo \#21) (figura 5). La morfología general de las cuentas es discoidal, con orificio central. Dentro de esta morfología general, la mayoría de las piezas tiene una morfología subcircular, mientras que algunas presentan una morfología subpentagonal. Por su parte la morfología de los tembetás es cilíndrica alargada, con un extremo en forma de mamelón o "botón".

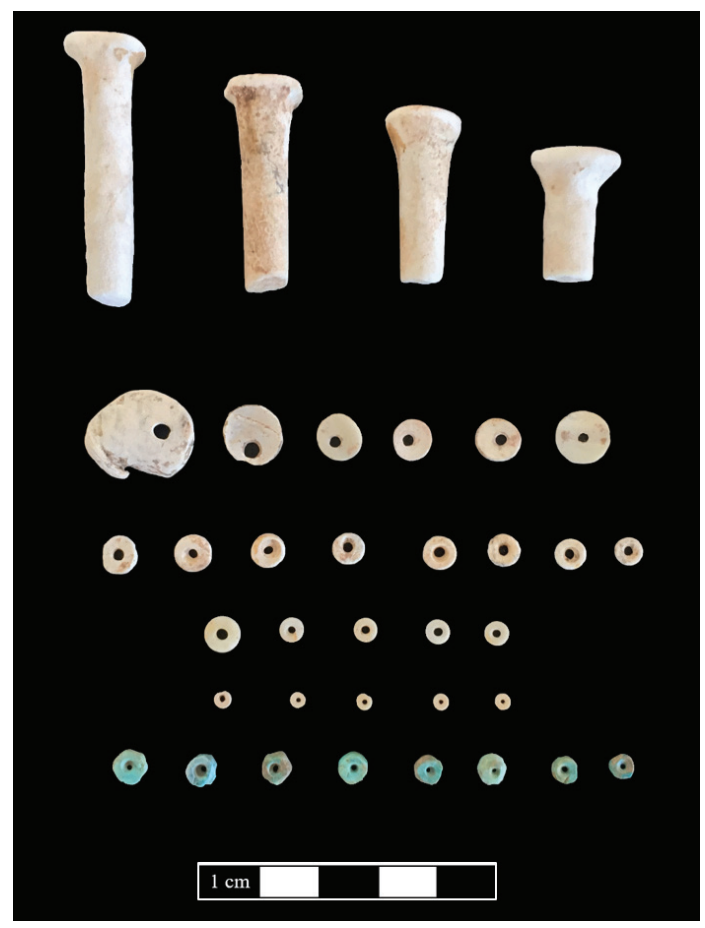

Figura 5. Ornamentos corporales en CS-1 (se presenta una selección de estos) 
Los grupos de cuentas pueden subdividirse en tres tipos, según sus materias primas: 1) cuentas producidas en materia prima lítica carbonáticas $(n=567), 561$ piezas producidas usando una materia prima lítica carbonática terrosa y 6 piezas producidas usando una materia prima carbonática vítrea; 2) cuentas producidas en material malacológico nacarado $(n=587)$; y 3$)$ cuentas producidas en materia prima lítica turquesa $(n=62)$. Se registró además un grupo de cuentas (\#19) de materias primas líticas mixtas $(n=27)$ tanto carbonática como turquesa.

Todos los tembetás fueron producidos usando una materia prima lítica carbonática. Posiblemente esto se deba a dos factores: a) que dicha materia prima sea más blanda que las otras, facilitando la formatización de un artefacto de morfología comparativamente compleja en tanto que requiere la talla de un cilindro con terminación en un botón redondeado; b) que el tamaño de los guijarros o bloques de esta materia prima fueran adecuados para la extracción de un ornamento de proporciones longilíneas como lo son los tembetás.

El estado general de conservación del conjunto de cuentas es bueno ya que el $20 \%$ de la muestra corresponde a piezas totalmente enteras y el $37 \%$ de la muestra incluye piezas enteras y solo algunos fragmentos. Los 16 tembetás se encuentran aparentemente enteros.

Los datos sobre tamaño indican que las cuentas registran diámetros mínimos de $2 \mathrm{~mm}$ y máximos de $18 \mathrm{~mm}$, siendo los más frecuentes los de $5 \mathrm{~mm}$ (registrados en 8 grupos), seguidos por diámetros de $6,7,8$ y $9 \mathrm{~mm}$.

El tamaño de los tembetás muestra una alta estabilidad en el diámetro de su porción cilíndrica (entre 7 u $8 \mathrm{~mm}$ ), mientras que la superficie de la porción del botón (calculada mediante la multiplicación de largo por ancho) y el largo del cilindro son variables. Esto sugiere que el diámetro era estandarizado debido a la superficie de penetración que debía generarse en la piel del sujeto para atravesarla y suspenderlo, mientras que la superficie del botón y el largo posiblemente podrían depender de otros factores, tales como la edad, el género y/o la contextura física-facial de la persona, que requirieran largos mayores o menores.

Dadas las condiciones de registro de estos materiales, en el contexto de una excavación de rescate, su potencial asociación con los restos humanos no está documentada, por lo cual es imposible realizar inferencias respecto de la vinculación de los ornamentos con el género y edad de las personas enterradas. Tampoco es posible indicar que todos los individuos hayan sido enterrados ornamentados con cuentas. Sin embargo, es interesante notar que la cantidad de tembetás $(\mathrm{N}=16)$ es menor que el número mínimo de individuos $(\mathrm{NMI}=24)$, por lo cual es posible sugerir que no todos ellos/ellas portaban este tipo de ornamento, lo cual, a su vez, implica algún tipo de diferenciación social interna al grupo de personas enterradas.

\section{Puntas de proyectil}

De la colección generada en los trabajos de campo en CS-1 se obtuvieron ocho artefactos formatizados correspondientes, en su totalidad, al grupo tipológico de las puntas de proyectil (tabla 2; figura 6). La materia prima más frecuente utilizada en su confección corresponde a las rocas silíceas $(75 \%)$, de calidad muy buena para la talla, excepto la correspondiente a la pieza \#CS/91-5, que es de calidad excelente. También se registró una vulcanita (de calidad buena-muy buena) y una obsidiana (de calidad muy buena-excelente). Esta última procede, según su análisis geoquímico realizado por Fluorescencia de Rayos X (XRF) en el MURR (Estados Unidos), de la fuente de obsidiana denominada El Peceño, localizada a aproximadamente $70 \mathrm{~km}$ al suroeste de CS-1, por lo que es considerada como no local. Las demás rocas son de procedencia local (sensu Civalero y Franco 2003). Ninguna de las puntas de proyectil presenta reserva de corteza. El 62,5\% de ellas se halla en estado fragmentado (tabla 2; figura 6). Las ocho puntas de proyectil fueron confeccionadas bajo la técnica de manufactura por presión. Los lascados son continuos y 
profundos a lo largo del borde perimetral de cada una de las puntas de proyectil. La forma perimetral del limbo de mayor representación es la triangular alargada (tabla 2). La forma secundaria del borde del limbo es mayoritariamente convexa. La serie técnica de la mayoría de estas piezas implica retalla, retoque y microretoque extendidos. Todas presentan sección transversal biconvexa, excepto la pieza $\mathrm{N}^{\circ} \# \mathrm{CS} / 91-3$, que es triangular redondeada. Ninguna presenta evidencias de reactivación y la mayoría (75\%) son apedunculadas, excepto en los casos que se clasifican como fragmentos no diferenciados por poseer fracturas en el extremo basal-proximal. El 50\% de las puntas de proyectil apedunculadas posee un tratamiento técnico diferencial de la base de tipo normal y, en la otra mitad, se observa adelgazamiento por retoques. El tratamiento del ápice y la forma de la base del limbo se describen en la tabla 2.

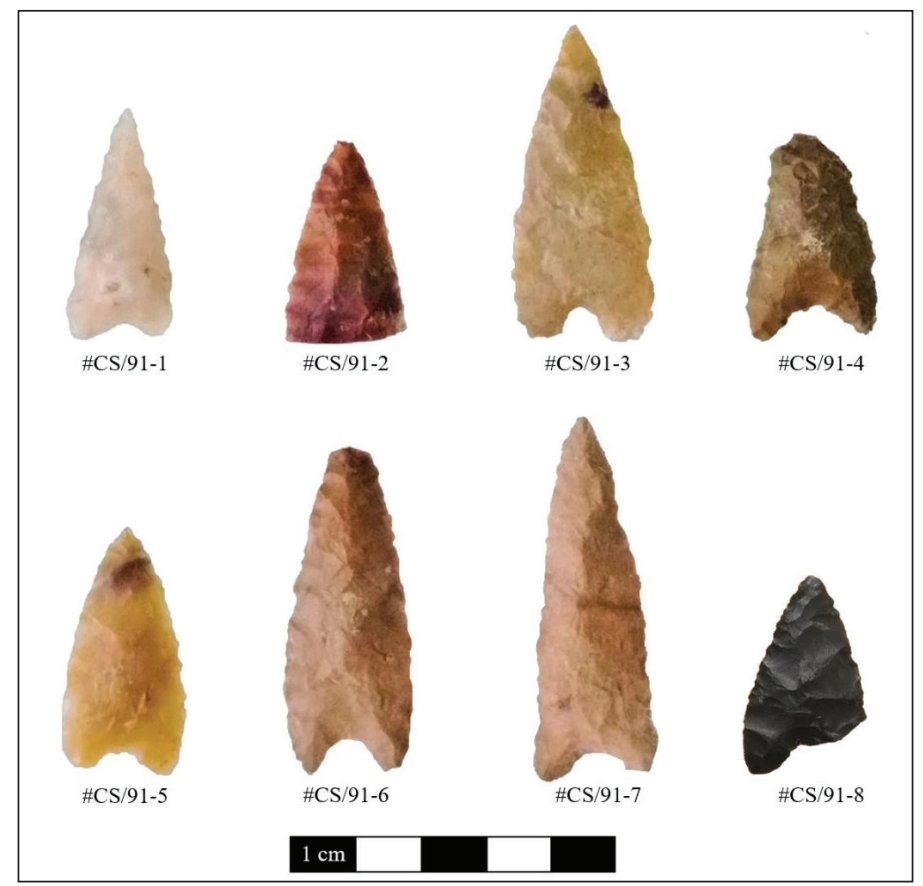

Figura 6. Instrumentos líticos en CS-1

Tabla 2. Variables morfométricas de las puntas de proyectil registradas en CS-1 Nota general: Largo, Ancho y Espesor en mm; Peso en g.

\begin{tabular}{|l|c|c|c|c|c|c|c|c|c|c|}
\hline Código & MP & Estado & Largo & Ancho & Espesor & Peso & $\begin{array}{c}\text { Apice } \\
\text { for }\end{array}$ & $\begin{array}{c}\text { For } \\
\text { Limbo }\end{array}$ & $\begin{array}{c}\text { Base } \\
\text { limbo }\end{array}$ & Sub-G Tip \\
\hline CS/91-1 & Sílice & Entero & 36 & 15 & 5 & 2,4 & Normal & $\begin{array}{c}\text { Triangular } \\
\text { alargada }\end{array}$ & $\begin{array}{c}\text { Cóncava } \\
\text { atenuada }\end{array}$ & $\begin{array}{c}\text { Pdp } \\
\text { Apedunculada }\end{array}$ \\
\hline CS/91-2 & Sílice & Fracturado & 31 & 17 & 5 & 2,7 & $\begin{array}{c}\text { No } \\
\text { aplica }\end{array}$ & $\begin{array}{c}\text { Triangular } \\
\text { alargada }\end{array}$ & No aplica & $\begin{array}{c}\text { Pdp Frag. No } \\
\text { Dif }\end{array}$ \\
\hline CS/91-3 & Sílice & Entero & 47 & 22 & 5 & 5,2 & Normal & $\begin{array}{c}\text { Triangular } \\
\text { alargada }\end{array}$ & $\begin{array}{c}\text { Cóncava } \\
\text { profunda }\end{array}$ & $\begin{array}{c}\text { Pdp } \\
\text { Apedunculada }\end{array}$ \\
\hline CS/91-4 & Sílice & Fracturado & 33 & 20 & 6 & 4,0 & $\begin{array}{c}\text { No } \\
\text { pplica }\end{array}$ & $\begin{array}{c}\text { C/ } \\
\text { asimetría } \\
\text { bilateral }\end{array}$ & $\begin{array}{c}\text { Cóncava } \\
\text { atenuada }\end{array}$ & $\begin{array}{c}\text { Pdp } \\
\text { Apedunculada }\end{array}$ \\
\hline
\end{tabular}


Relaciones de la Sociedad Argentina de Antropología 46 (2), julio-diciembre 2021: 561-594

(Tabla 2. Continuación)

\begin{tabular}{|l|c|c|c|c|c|c|c|c|c|c|}
\hline Código & MP & Estado & Largo & Ancho & Espesor & Peso & $\begin{array}{c}\text { Apice } \\
\text { for }\end{array}$ & $\begin{array}{c}\text { For } \\
\text { Limbo }\end{array}$ & $\begin{array}{c}\text { Base } \\
\text { limbo }\end{array}$ & Sub-G Tip \\
\hline CS/91-5 & Sílice & Entero & 38 & 18 & 5 & 3,3 & Normal & Lanceolada & $\begin{array}{c}\text { Cóncava } \\
\text { atenuada }\end{array}$ & $\begin{array}{c}\text { Pdp } \\
\text { Apedunculada }\end{array}$ \\
\hline CS/91-6 & Sílice & Fracturado & 51 & 20 & 5 & 5,2 & $\begin{array}{c}\text { No } \\
\text { aplica }\end{array}$ & $\begin{array}{c}\text { C/ } \\
\text { asimetría } \\
\text { bilateral }\end{array}$ & $\begin{array}{c}\text { Cóncava } \\
\text { profunda }\end{array}$ & $\begin{array}{c}\text { Pdp } \\
\text { Apedunculada }\end{array}$ \\
\hline CS/91-7 & Vulcanita & Fracturado & 55 & 18 & 6 & 5,5 & Normal & $\begin{array}{c}\text { Triangular } \\
\text { alargada }\end{array}$ & $\begin{array}{c}\text { Cóncava } \\
\text { profunda }\end{array}$ & $\begin{array}{c}\text { Pdp } \\
\text { Apedunculada }\end{array}$ \\
\hline CS/91-8 & Obsidiana & Fracturado & 36 & 18 & 5 & 2,5 & Normal & Lanceolada & No aplica & $\begin{array}{c}\text { Pdp Frag. No } \\
\text { Dif }\end{array}$ \\
\hline
\end{tabular}

Referencias: Ápice for= Forma/tratamiento del ápice; For Limbo= Forma perimetral del limbo; Base limbo= Forma de la base del limbo; Sub-G Tip= Subgrupo tipológico.

\section{Registro zooarqueológico}

Los trabajos de campo permitieron recuperar 496 especímenes óseos no humanos que fueron identificados siguiendo criterios previamente definidos en la región (Neme y Gil 2002). No se contabilizaron los especímenes de moluscos de las cuentas de collar. La tabla 3 señala que toda la fauna identificada corresponde al grupo de los mamíferos, confirmando el registro de cánidos y roedores. Las especies identificadas son típicas del ambiente en el que se encuentra el sitio, con excepción de Lycalopex culpaeus (y probablemente Mollusca). Es notoria la abundancia de cánidos (Lycalopex sp.) respecto a los otros taxones, incluso considerando su baja presencia en el registro zooarqueológico regional (Neme y Gil 2002; Otaola et al. 2012). Asimismo, se destaca el registro de L. culpaeus fuera de su actual área de distribución geográfica (Pía et al. 2019). En el apartado de resultados de isótopos estables se profundiza sobre el significado de los restos de Lycalopex sp. en el contexto de CS-1.

Ninguno de los taxones presenta evidencias de consumo humano. Los microvertebrados, además, presentan evidencias de digestión por aves rapaces de la categoría 2-3 de modificación (Athene cunicularia o Bubo virginianus), por lo que se asume que su presencia en el sitio no es de origen antrópico. La meteorización de los especímenes óseos es baja (0 y 1), con muchas evidencias de marcas de raíces y precipitaciones de óxido de manganeso.

Tabla 3. Taxa recuperados en CS-1, abundancia medida en NISP

\begin{tabular}{|c|c|}
\hline Taxa & NISP \\
\hline Mamífero & 2 \\
\hline Mamífero Pequeño & 9 \\
\hline Mamífero mediano & 20 \\
\hline Canidae indet. & 3 \\
\hline Lycalopex sp. & 4 \\
\hline Lycalopex culpaeus & 64 \\
\hline Lycalopex griseus & 10 \\
\hline Galea leucoblehara & 2 \\
\hline
\end{tabular}


(Tabla 3. Continuación)

\begin{tabular}{|c|c|}
\hline Taxa & NISP \\
\hline Microcavia australis & 65 \\
\hline Caviidae indet & 4 \\
\hline Tympanoctomys barrerae & 12 \\
\hline Akodon dolores & 6 \\
\hline Graomys griseoflavus & 6 \\
\hline Oligoryzomys flavescens & 1 \\
\hline Oligoryzomys sp. & 1 \\
\hline Mollusca & 1 \\
\hline Indeterminados & 286 \\
\hline Total & 496 \\
\hline
\end{tabular}

Isótopos estables $\left(\delta^{13} \mathrm{C}, \delta^{15} \mathrm{~N}\right.$ y $\left.\delta^{18} \mathrm{O}\right)$

Para los humanos se obtuvo información isotópica sobre 24 individuos cuyos datos de sexo y edad están detallados en la tabla 4. Asimismo, se obtuvo un valor sobre colágeno de fauna ( $L y$ calopex sp.) que es presentado separadamente, aunque en relación con los resultados obtenidos para los humanos de CS-1. Para entender las tendencias en las dietas humanas inferidas desde los isótopos estables se requiere un modelo sobre las cadenas tróficas que contemple la variabilidad isotópica de los recursos de base (Szpak et al. 2013). Por ello se utilizaron los datos disponibles en la región (Gil et al. 2006, 2014; Giardina et al. 2014; Otaola et al. 2018).

$\delta^{13} \mathrm{C}, \delta^{15} \mathrm{~N}$ y dieta humana en $\mathrm{CS}-1$

Para las muestras humanas se obtuvo un valor de $\delta^{13} \mathrm{C}$ promedio de $-14,3 \%_{0}$ ( $\mathrm{DS}=1,5$, rango de $-10,3 \%$ a $-15,9 \%$ o) en colágeno y de $-9,1 \%$ o ( $\mathrm{DS}=1,7$, rango de $-4,4 \%$ o $-10,8 \%$ o) en bioapatita. Esto indica una diferencia promedio de 5,26\%o entre el $\delta^{13} \mathrm{C}$ de bioapatita y colágeno. Por su parte el promedio de $\delta^{15} \mathrm{~N}$ es de $11,8 \%$ ( $\mathrm{DS}=1,27$, rango de $15,3 \%$ a $10,4 \%$ ) mientras que el $\delta^{18} \mathrm{O}$ es de $-5,5 \%$ ( $\mathrm{DS}=2,2$, rango de $-1,3 \%$ a $-7,9 \%$ ). La figura 7 presenta la tendencia en $\delta^{13} \mathrm{C}_{\mathrm{col}}$ y $\delta^{15} \mathrm{~N}$ mostrando por una parte el conjunto total y por el otro segregando por edad: adultos y subadultos. Segregados por edad, los individuos subadultos presentan valores más altos en todos los isótopos y significativamente diferentes en cuanto a $\delta^{13} \mathrm{C}$. Se registra una baja variabilidad interindividual, principalmente en el grupo adulto, cuyo análisis se profundiza en el resto de esta sección. Para complementar y tener un mayor acercamiento se aplican a continuación un conjunto de herramientas interpretativas detalladas en la parte metodológica.

La figura 8a compara los valores correspondientes a la fracción proteica de los individuos de CS-1 en referencia a la ecología isotópica de la región, conformada a partir de los recursos más significativos para la dieta humana de acuerdo con información arqueológica de base (Gil y Neme 2010; Giardina et al. 2015; Otaola et al. 2018). Considerando los valores de los recursos corregidos por fraccionamiento isotópico, se destaca la proximidad de los humanos principalmente con respecto al guanaco, tanto en términos de $\delta^{15} \mathrm{~N}$ como de $\delta^{13} \mathrm{C}_{\text {col }}$. El valor promedio de los individuos de CS-1 correspondiente a $\delta^{13} \mathrm{C}_{\text {apa }}$ (figura 8b) se ubica dentro de los límites definidos por los valores de guanaco, armadillo y peces, por lo que la dieta total podría componerse princi- 


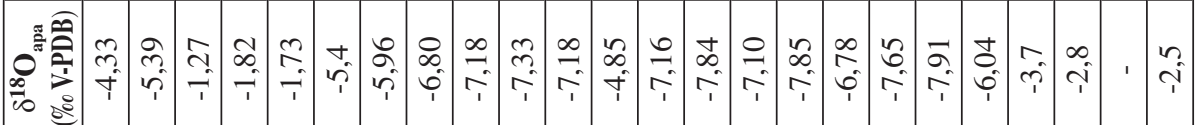

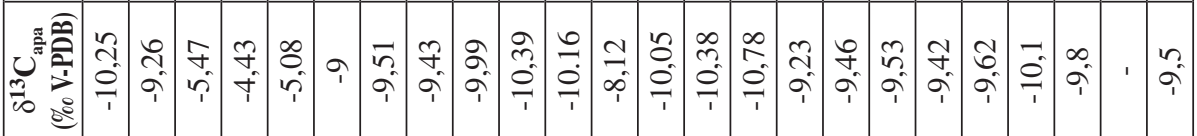

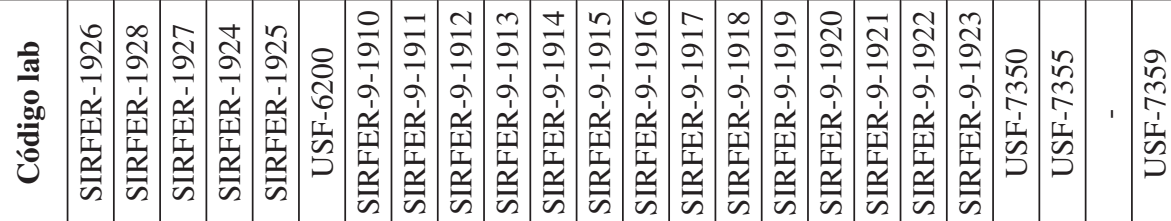

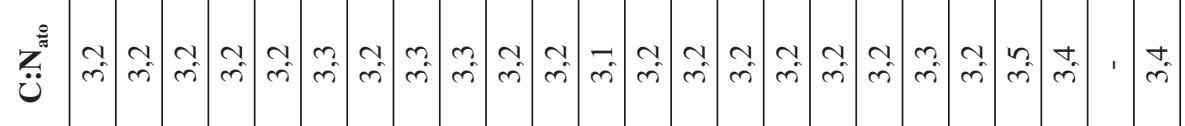

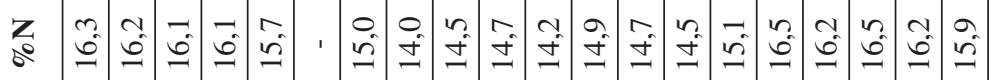

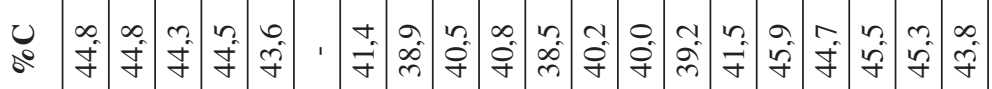

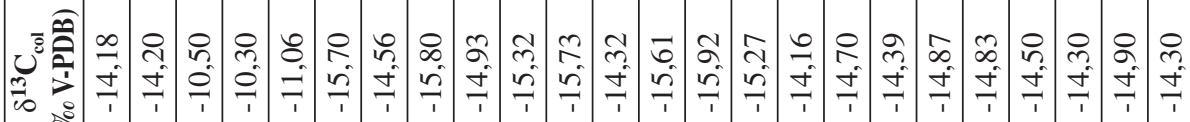

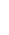

z

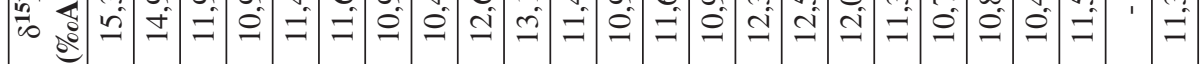

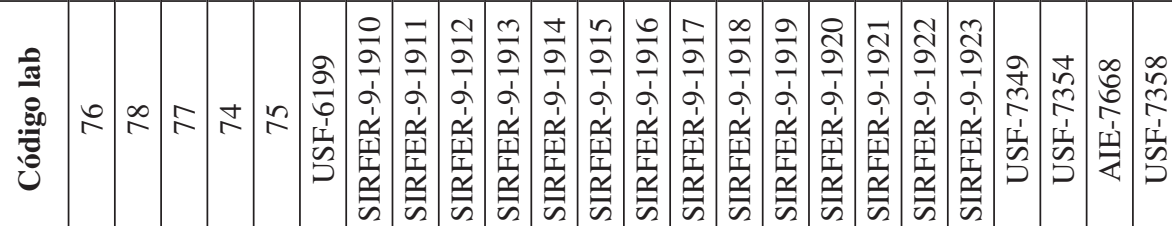

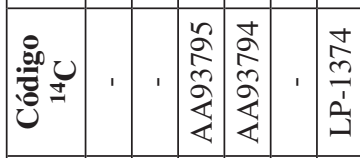

喜

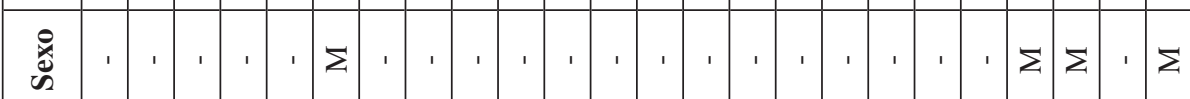

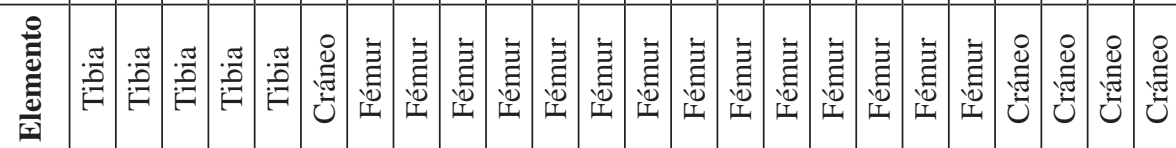

: 


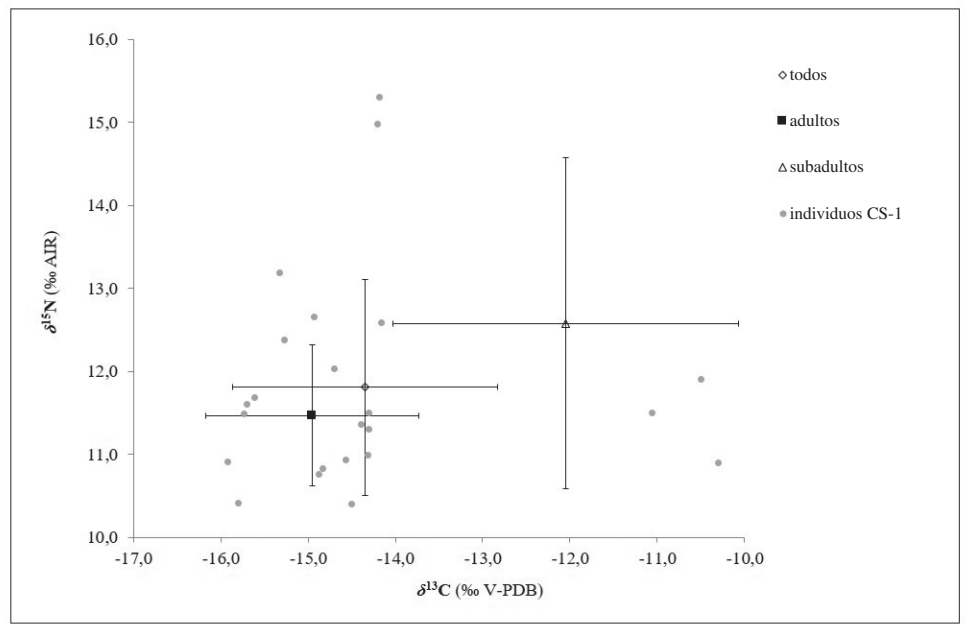

Figura 7. Promedios y desvíos estándar en las muestras de CS-1, haciendo la distinción entre adultos y subadultos

palmente por estos recursos. En las figuras 8c y 8d se comparan los valores de colágeno y bioapatita obtenidos sobre hueso humano con respecto a los recursos corregidos con los fraccionamientos propuestos por Fernandes (2016). Con estas correcciones se observa cierta correspondencia en los valores de $\delta^{15} \mathrm{~N}$ de CS-1 y los de guanaco, reídos (huevo y carne) y peces. Asimismo, los valores de $\delta^{13} \mathrm{C}_{\text {col }}$ y $\delta^{13} \mathrm{C}_{\text {apa }}$ de CS-1 se encuentran en una posición intermedia entre los peces y los reídos, coincidiendo en mayor medida con el de los armadillos. Mediante estas aproximaciones bivariadas, se destaca una participación de recursos proteicos más o menos constante en la dieta, como en el caso del armadillo, con ciertas variaciones en cuanto al papel del guanaco, los peces y los reídos, dependiendo del fraccionamiento considerado. El maíz y el algarrobo, por otra parte, se mantienen alejados de CS-1, con uno y otro grupo de fraccionamientos.

Los modelos de mezcla de dieta basados en estadística bayesiana indican distintos escenarios según se incluya guanaco, huevo de reídos y/o peces: el escenario $A$ incluye guanaco, armadillo, maíz y algarrobo; el $B$, guanaco, armadillo, maíz, algarrobo y huevo; el $C$, guanaco, armadillo, maíz, algarrobo y pescado; el $D$, armadillo, maíz, algarrobo y pescado; el $E$, guanaco, armadillo, maíz, algarrobo, pescado y huevo; y el $F$, armadillo, maíz, algarrobo y huevo (figura 9). En todos los escenarios el guanaco constituye cerca del $20 \%$ de la dieta, con una mediana que alcanza el $24 \%$ como máximo y $13 \%$ como mínimo; el armadillo forma parte de la dieta en un menor porcentaje (menos del 20\%) con una mediana que alcanza un valor máximo de $27 \%$ y un valor mínimo de $10 \%$. Las contribuciones del maíz y el algarrobo se encuentran entre las más altas, con sus respectivas medianas rondando el $30 \%$ y el $20 \%$. Solo en el escenario que incluye todos los recursos simultáneamente y aquel que no incluye ni guanaco ni peces, la mediana de la contribución del maíz supera el 30\% (tabla suplementaria 3: http://doi.org/10.5281/ zenodo.4769808). Cabe aclarar que estos dos escenarios son los que presentan mayor debilidad, en un caso por incluir un número elevado de recursos en la misma modelización y en el otro por no incluir dos de las fuentes de proteína más relevantes de acuerdo con el registro arqueológico. Finalmente, la contribución del pescado y del huevo fue mucho menos importante que la del guanaco y del armadillo, con medianas que no alcanzan el 20\%. Al considerar los recursos silvestres respecto al maíz, se observa en todos los modelos una predominancia del primer grupo respecto al segundo. 

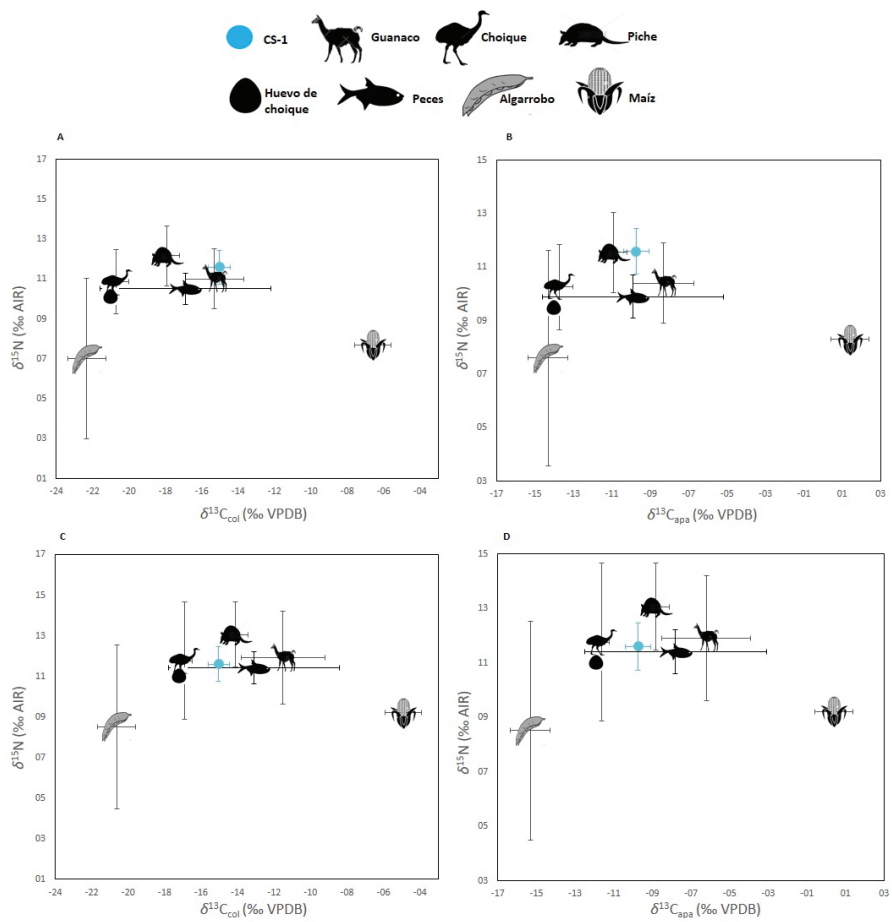

Figura 8. A) y B) Tendencias isotópicas $\left(\delta^{13} \mathrm{C}_{\text {col }}, \delta^{15} \mathrm{~N}, \delta^{13} \mathrm{C}_{\text {apa }}\right)$ de las muestras óseas humanas (adultos) respecto de los recursos de la región del Atuel (corregidos por discriminación isotópica de acuerdo con Bocherens y Druker 2003; Tykot et al. 2009; Cheung y Szpak 2020; Gil et al. 2020). C y D) Tendencias isotópicas de las muestras óseas humanas respecto de los recursos (corregidos por discriminación isotópica de acuerdo con Fernandes 2016)

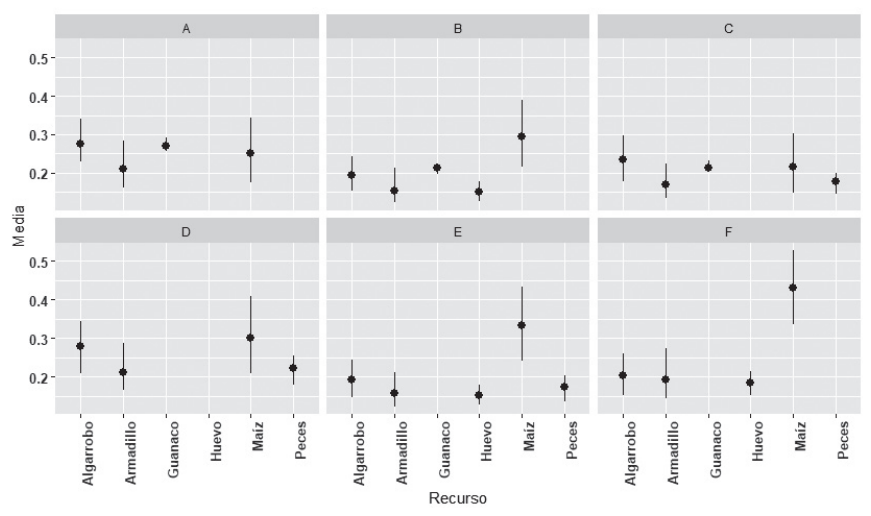

Figura 9. Medias y desvíos de los porcentajes de contribución calórica de los diferentes recursos de acuerdo con el software FRUITS. Las medias y desvíos se calcularon a partir de los valores medios de cada recurso para cada individuo. 
$\delta^{18} \mathrm{O}$ y movilidad humana en CS-1

La figura 10 muestra las tendencias geográficas para los valores de $\delta^{18} \mathrm{O}$ de las aguas superficiales junto con los valores de los humanos de CS-1 corregidos (Gil et al. 2012; Ugan et al. 2012). Los valores de los individuos varían entre -7,4\% y -11,3\%o, y muestran el uso de un amplio rango de fuentes (tabla 4, figura 10). Se diferencian dos patrones: uno en el que los individuos se asocian al área del Cerro Nevado y otro en el que se asocian a dicha área y a la de los ríos Atuel/ Diamante. Para este último puede también sugerirse el uso de otros sectores cuyos valores de $\delta^{18} \mathrm{O}$ aún se desconocen (figura 1 ). Dado que los valores no muestran un uso predominante de una fuente cercana al sitio, se debilita la posibilidad de que los individuos de CS-1 mantuvieran una residencia a largo plazo, asociada a una baja movilidad residencial cercana al sedentarismo.

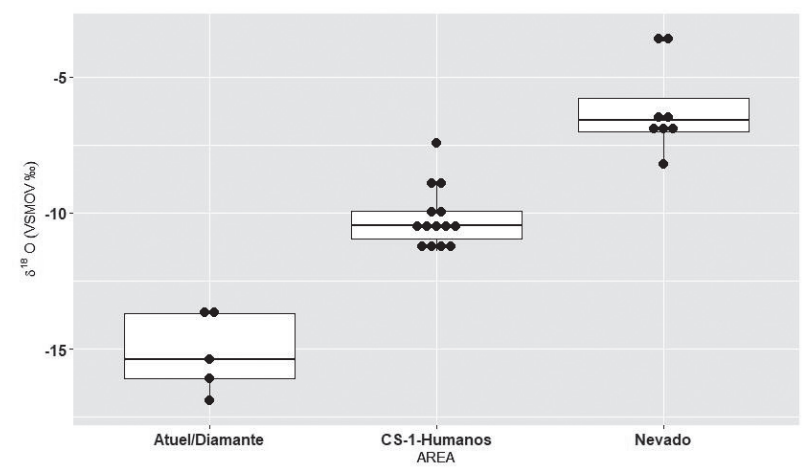

Figura 10. Valores de $\delta^{18} \mathrm{O}$ de aguas y su comparación con los valores de éste isótopo en humanos

Valores de $\delta^{13} \mathrm{C}, \delta^{15} \mathrm{~N}$ obtenidos en Lycalopex sp.

Un hueso asignado a Lycalopex cf. L. culpaeus fue procesado para medir los valores en las relaciones de isótopos estables de carbono y nitrógeno en colágeno. Se realizó sobre un radio-cúbito y su resultado fue para $\delta^{13} \mathrm{C}$ de $-12,4 \%$ y para $\delta^{15} \mathrm{~N}$ de 12,6\%o (Código MSR\#651, relación $\mathrm{C}: \mathrm{N}$ atómica $=3,2$ ). Al comparar dichos resultados con los de otros carnívoros nativos (Otaola et al. 2018) y los humanos de CS-1 se destacan los siguientes aspectos. Por una parte, los valores de ambas relaciones isotópicas obtenidos en la muestra de Lycalopex sp. de CS-1 se diferencian significativamente de los de carnívoros previamente analizados en la región $(\mathrm{n}=17)$. Lycalopex sp. de CS-1 tiene ambos valores significativamente más altos, entre 4 y 5 puntos para $\delta^{15} \mathrm{~N}$, y entre 3 y 6 puntos para $\delta^{13} \mathrm{C}$ (figura 11). Por otra parte, dichos valores se asemejan notablemente a los humanos del mismo sitio. La diferencia respecto al promedio de $\delta^{13} \mathrm{C}$ en humanos es de $1,9 \%$ o y respecto del promedio de $\delta^{15} \mathrm{~N}$ es de $0,8 \%$. Como muestra la figura 11 , la dieta inferida para el carnívoro de CS-1 se asemeja en mayor medida a la de los humanos de CS-1 que a la de los otros carnívoros nativos. 


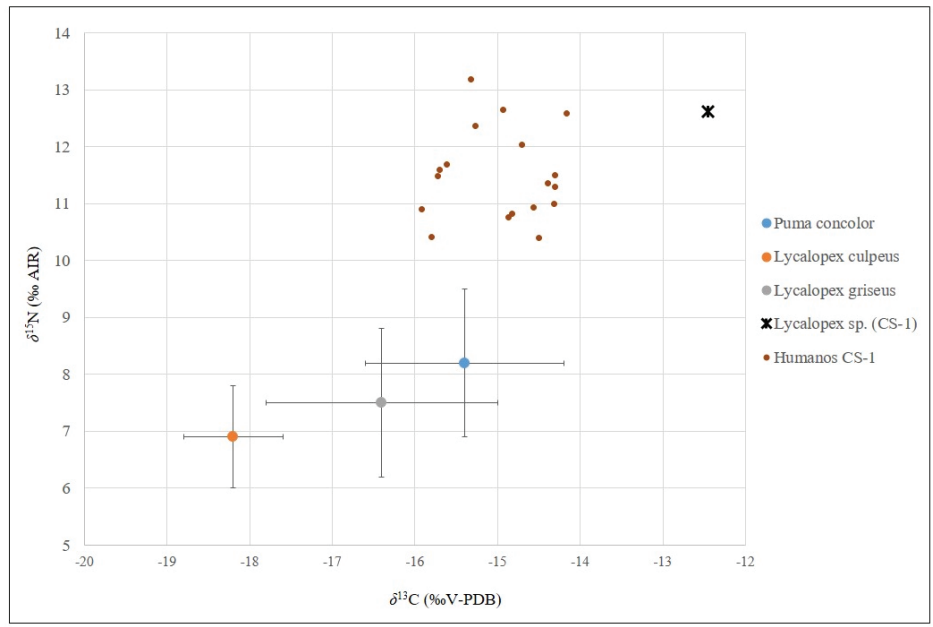

Figura 11. Valores de $\delta^{13} \mathrm{C}$ y $\delta^{15} \mathrm{~N}$ de carnívoros autóctonos de la región en comparación con el de Lycalopex sp. recuperado en CS-1

\section{CAÑADA SECA-1: REGISTRO ARQUEOLÓGICO DE POBLACIONES DE PEQUEÑA ESCALA}

El sitio CS-1 se emplaza en una región límite para el registro de plantas domésticas prehispánicas en la vertiente oriental de los Andes (Lagiglia 1982; Gil et al. 2014). Su cronología es unos 500 a 800 años posterior a la incorporación de plantas domésticas en el registro arqueológico regional. De acuerdo con su temporalidad y con la historia cultural regional, se lo asignó a comunidades agroalfareras afines a Agrelo, cultura que ha sido clásicamente asociada a un contexto de agricultores con baja movilidad residencial (Lagiglia 1991; Bárcena 2001).

Los resultados de isótopos estables muestran que, si bien los humanos presentan valores relativamente altos de $\delta^{13} \mathrm{C}$, estos no se explican de manera exclusiva por una mayor importancia del maíz en la dieta. Los recursos silvestres, faunísticos principalmente, también incluyen una señal $\mathrm{C}_{4}$ que sería trasmitida a los humanos (Otaola et al. 2018; Gil et al. 2020). Asimismo, al modelizar diferentes combinaciones de recursos empleando el software FRUITS, se observa en general una importancia media a baja del maíz, con frecuencias semejantes para cada recurso silvestre. En términos absolutos, el aporte de los recursos silvestres a la dieta sobrepasa al de los recursos domésticos. La baja importancia del maíz posibilitaría la reversibilidad desde una organización apoyada en la producción de alimentos a una basada en la extracción de recursos silvestres, cuando las condiciones del sistema humano-ambiental sufren algún cambio (véase Gil et al. 2020 para un ejemplo de los últimos 1000 años en una macro región que incluye el valle del Atuel).

En comparación con los resultados obtenidos con el modelo MixSIAR (Gil et al. 2018) a partir de cinco grupos de recursos (guanaco, armadillo, maíz, algarrobo y pescado), se destaca la menor contribución del armadillo a la dieta humana y se observa que la importancia del maíz y el algarrobo es relativamente mayor a la de la proteína animal considerada individualmente. También es interesante que se mantiene la importancia levemente superior del algarrobo en relación con el maíz y la del guanaco con respecto al pescado. La aplicación de este modelo y la posibilidad de considerar los aportes de cada recurso en términos de proteína y energía (lípidos y carbohidratos), pudo haber contribuido a refinar algunas tendencias observadas previamente en la dieta de este 
conjunto. Es interesante notar que una de las contribuciones que más varió en importancia es la del armadillo, cuyo aporte de lípidos es considerablemente mayor al del resto de los recursos, variable que pudo ser incorporada a través del FRUITS. Asimismo, aunque la importancia relativa del maíz continúa siendo baja, es mayor que la observada mediante MixSIAR, lo que también podría responder a que se están considerando las concentraciones de proteína y energía de los recursos de manera particular.

En cuanto a la movilidad, al considerar los valores de $\delta^{18} \mathrm{O}$ se descarta una residencia de largo plazo en este sector del valle. La integración de estos datos con algunas observaciones realizadas sobre el patrón de entierro y el análisis del acompañamiento mortuorio, apunta a descartar la idea de un grupo humano con una baja movilidad. Por una parte, la modalidad de entierro secundaria estaría indicando un transporte de los individuos inhumados desde otro sector. Si bien la información del contexto de inhumación es muy escasa y limita nuestras interpretaciones, la modalidad de entierro secundario puede inferirse si se tienen en cuenta otros antecedentes regionales (Novellino 2002; Gil y Neme 2010; Peralta 2019), como también si se consideran algunas particularidades del registro: la presencia de pigmento ocre en algunos elementos y la disociación de elementos anatómicos pueden ser indicativas del armado de paquetes funerarios característicos de los entierros secundarios (Luna 2008). En este mismo sentido, aunque las marcas antrópicas no modernas se registraron en un muy bajo porcentaje, la mayoría se restringen al postcráneo (huesos largos de miembros inferiores) y se corresponden a un único tipo (cutmarks), lo que podría asociarse con el descarne en contextos de entierro secundario (Buikstra y Ubelaker 1994; White y Folkens 2005).

Por otra parte, la gran cantidad de tembetás y cuentas asociadas a los entierros muestran una importante diversidad de técnicas de confección y de materias primas, algunas de las cuales podrían haber sido obtenidas de lugares distantes, como la turquesa o los especímenes de moluscos (Berón 2007; Lagiglia 2009). Si bien no se pudo realizar una identificación taxonómica a partir del registro de cuentas malacológicas, se estima que tendrían un origen marino. Esta idea se sustenta por la ausencia de gasterópodos o bivalvos locales con una estructura adecuada para este tipo de trabajo y por los antecedentes regionales que corroboraron la presencia de moluscos marinos, muy probablemente del Pacífico (Neme y Gil 2005; Lagiglia 2009). Tampoco se han registrado moluscos ni piezas de materias primas líticas formatizados en el sitio, lo cual implica que estos fueron ingresados como ornamentos e instrumentos ya manufacturados, potencialmente usados por los individuos en vida y/o como ajuar de acompañamiento mortuorio. Determinar si la presencia de estos ornamentos implica aprovisionamiento directo o intercambio en un sistema macrorregional, involucrará futuros abordajes más exhaustivos. De igual forma, en vista de la evidencia, los rangos de acción de estos grupos humanos serían mucho más amplios que lo sugerido por las interpretaciones iniciales.

Sumado a esto, las tendencias obtenidas del análisis tecno-tipológico de las puntas de proyectil de CS-1 señalan un conjunto donde predominan las rocas locales de muy buena calidad para la talla, y donde la obsidiana (no local) habría sido obtenida a partir de un aprovisionamiento de tipo cíclico directo, como propuso Salgán (2015) para otra área del sur de Mendoza. Dicho tipo de abastecimiento implica que las rocas son obtenidas directamente en la fuente, ya sea porque los rangos de acción de las poblaciones son amplios o porque se dan incursiones logísticas a largas distancias (Salgán 2015), sin que sea necesaria la interacción con otros grupos. Este tipo de aprovisionamiento lítico se observó también en el sitio de actividades múltiples Los Gallegos 1, localizado a unos $10 \mathrm{~km}$ de CS-1 y con una cronología que también corresponde al Holoceno tardío final (Sugrañes et al. 2020).

Los resultados obtenidos en este trabajo mostraron una dieta en la que los recursos vegetales domesticados estuvieron presentes, aunque con una importancia variable. A pesar de conocerlos y haberlos incorporado en alguna medida, el rol de estos recursos no habría impactado de 
manera significativa en las estrategias de subsistencia y movilidad, que se mantuvieron ligadas mayormente a la caza-recolección. Al integrar CS-1 con sitios próximos espacial y temporalmente, como Rincón del Atuel-1 (RA-1) y Jaime Prats-1 (JP-1) (Gil et al. 2018), se destaca que durante los primeros 1000 años posteriores a la incorporación de cultígenos, la subsistencia no se organizó de manera estable y exclusiva en torno a la producción de alimentos, sino que se habría logrado una suerte de integración entre la estrategia productiva y la estrategia cazadora-recolectora predominante. Esta alta variabilidad cuestiona el uso de unidades estilísticas que homogenicen los procesos humanos.

\section{Consideraciones sobre los restos de cánido hallados en CS-1}

La fauna asociada no incluye a las principales especies explotadas para la subsistencia humana como guanaco, armadillo, reídos o huevos de reídos (Gil y Neme 2010). Sin tener en cuenta las especies cuya presencia no se relaciona de manera directa con la actividad humana en el pasado, el registro de fauna se limita a carnívoros no domésticos representados por dos especies. Una de estas habita en la actualidad el área, L. griseus, mientras que la otra, L. culpaeus, lo hace en sectores andinos patagónicos (Roig 1965). Sumado a esto, se destaca la gran abundancia de restos de estos carnívoros, que es única en la región (Otaola et al. 2018; López et al. 2019). Se propone que fueron intencionalmente depositados por los humanos en las inhumaciones. Las mediciones de relaciones de isótopos estables de carbono y nitrógeno obtenidas son significativamente diferentes de las de otros carnívoros de la región y tienen una diferencia menor a $2 \%$ o con respecto a los valores de los humanos de CS-1. Esta diferencia se ha tomado como un umbral convencional para distinguir entre cánidos no domesticados y perros domésticos cuya dieta reflejaría la de los humanos con los que conviven (Guiry 2012).

Se destacan dos aspectos de esta evidencia. Por un lado, que efectivamente estos restos pertenezcan a un cánido doméstico, cuya depositación en contextos fúnebres ha constituido una práctica habitual (Byrd et al. 2013; Berón et al. 2015). La historia evolutiva y la fuerte hibridación de los carnívoros hacen dificultosas las identificaciones zooarqueológicas basadas en la comparación morfológica (Guiry 2012; Byrd et al. 2013; Popović et al. 2020). Por otra parte, puede plantearse que este patrón, comúnmente observado entre perros domésticos, también se haya dado con carnívoros silvestres. En esta línea, un reciente estudio molecular sobre restos registrados en Bolivia reclasificó especímenes previamente identificados como perro doméstico y los asignó a L. culpaeus (Popović et al. 2020). Según Prevosti et al. (2011), el cánido Dusicyon avus se habría extinguido en algún momento entre los ca. 3000-500 AP, por lo que la cronología y ubicación de CS-1 podría sugerir la presencia de este cánido en el sitio. Incluso Prates (2014), por ejemplo, reportó un entierro intencional de esta especie de zorro por parte de los seres humanos en un sitio de la región pampeana con cronologías dentro del Holoceno tardío, asociado a inhumaciones humanas. Sin embargo, los restos óseos de cánido recuperados en CS-1 son considerablemente más chicos en tamaño y su morfología sugiere alguna especie de Lycalopex, a pesar de haberlos recuperado en contextos de entierros humanos.

Si bien este hallazgo no tiene una implicancia directa para la discusión sobre la incorporación de cultígenos, sí aporta información valiosa e inédita con respecto a los antiguos ocupantes de CS-1 y a la complejidad de su desarrollo cultural y la relación con su entorno, que es, asimismo, relevante para entender las estrategias de subsistencia y movilidad particulares a este grupo humano. 


\section{CONCLUSIÓN: DIETA, MOVILIDAD Y TECNOLOGÍA CONDENSADAS EN UN MISMO ENTERRATORIO}

Desde el hallazgo de CS-1 transcurrieron varios años en los que los materiales de este sitio aportaron datos específicos de manera aislada, pero sin un detalle de su contexto y sin integrar la evidencia disponible. Los resultados obtenidos en este trabajo indican que el sitio CS-1 correspondió a un entierro múltiple compuesto por al menos veinticuatro individuos, incluyendo infantiles, juveniles y adultos. Las condiciones de recuperación de los esqueletos no fueron totalmente controladas, por lo que gran parte de la información contextual se perdió. Los fechados permiten estimar que sucedieron múltiples eventos inhumatorios, en los que pudieron existir entierros primarios y secundarios. Si bien estas condiciones no han permitido establecer asociaciones directas entre los individuos y los artefactos utilitarios (puntas líticas) y no utilitarios (ornamentos corporales) hallados en el sitio, ha sido posible evaluar que las puntas de proyectil fueron producidas sobre materias primas mayormente locales y están fragmentadas en alta proporción, sugiriendo la posibilidad de que fueran instrumentos utilizados en vida previamente a su depositación junto a las personas enterradas. Respecto de los ornamentos corporales (cuentas y tembetás), resulta importante destacar que estos pueden haber estado ornamentando el cuerpo y/o la ropa de las personas enterradas, aunque también pueden haber sido colocados como ofrenda asociada a ellas sin que la estuvieran portando en sus cuerpos al momento de ser enterrados, tema que no es posible dilucidar debido a que el contexto de recuperación mediante excavaciones de rescate carece de registros relevantes a este punto. Sin embargo, las materias primas de las cuentas y tembetás demuestran no solamente una cierta variabilidad interna en el uso de por lo menos tres tipos distintos de materiales para sus ornamentos (turquesa, carbonática y malacológica), sino también que por lo menos una fracción de estas -las de turquesa y las de moluscos posiblemente marinos- debe haber provenido de zonas de aprovisionamiento no local, lo cual a su vez permite inferir un amplio rango de acción de estas poblaciones. Por otra parte, si bien el alto número de cuentas potencialmente podría implicar un uso por y/o acompañamiento de todas las personas enterradas (sin poder diferenciar la existencia de tendencias por género o edad), la posibilidad de alguna diferenciación interna al grupo también existe en tanto que los tembetás son muchos menos que las personas enterradas.

Los resultados relativos a los isótopos estables muestran un patrón en CS-1 distinto al esperado para grupos sedentarios y agricultores. Se infiere variabilidad en la residencia de los individuos que al menos debilita la idea de un establecimiento local de largo plazo. Por su parte, la dieta incluiría un fuerte componente de recursos silvestres. El componente doméstico, presentado en términos de maíz, habría contribuido a la dieta en un 30\%, en promedio. Este registro puede interpretarse como resultado de una estrategia que si bien incluyó recursos domésticos fue distinta a la clásicamente caracterizada como agricultora y podría corresponderse mejor con la de productores de baja escala o cazadores-recolectores con cultígenos (Smith 2001; Winterhalder y Kennett 2020).

Los restos de fauna recuperados en el sitio destacan por la presencia de al menos dos especies de cánidos, uno de los cuales corresponde a una especie local (zorro gris), mientras que el otro correspondería a una especie proveniente de ambientes distantes (zorro colorado). Su significado en el sitio es difícil de explicar, pero es posible que hayan sido enterrados intencionalmente con los humanos. La presencia de estos ejemplares de fauna, junto con otras particularidades del sitio, como la presencia de maíz en algunas de las dietas, la ornamentación y el número de individuos, aportan sustento a la idea de un estilo de vida más complejo y versátil de lo que se ha interpretado comúnmente para los grupos de la región.

La comparación de CS-1 con sitios similares y próximos geográfica y temporalmente (JP-1 y RA-1), indica que es probable que se trate de ocupaciones correspondientes a un momento de retracción de la frontera agrícola meridional de tiempos prehispánicos. La alta variabilidad de 
estrategias en este sector del Atuel y del sur de Mendoza, genera la necesidad de entender cuáles son las causas para mantener un sistema en bajos niveles de producción y qué mecanismos enmarcaron esta reversión advertida en los estudios recientes (Gil et al. 2018, 2020).

\section{AGRADECIMIENTOS}

Las investigaciones se realizaron en el marco de los proyectos PICT 2016-2667 "Estrategias Humanas de Largo Plazo en Desiertos del Sur de Mendoza", PIP-CONICET 2015-20170342 "Estrategias Humanas y Variabilidad Ambiental en los Desiertos del Sur de Mendoza: Una Perspectiva Biogeográfica" y UNCuyo (Project 06/G805). Agradecemos a la dirección del Museo de Historia Natural de San Rafael por facilitar el estudio del sitio y al personal técnico de dicha institución por sus tareas de acondicionamiento y preservación. Agradecemos a Francisco Prevosti (CONICET) por corroborar y realizar identificaciones taxonómicas de los especímenes asignados a cánidos, y a Miriam I. Ayala por su asistencia y colaboración en el estudio de estos materiales. Agradecemos los aportes de dos evaluadores anónimos que realizaron comentarios y críticas muy valiosos que permitieron mejorar la versión previa. Un agradecimiento también para Sayuri Kochi por compartir su experiencia y saberes en el manejo de FRUITS, y a Darío Soria (IDEVEA-CONICET) por realizar el mapa para este trabajo.

\section{NOTAS}

1 Se conservó la definición de grupos de la colección. No se pudo precisar si las agrupaciones responden a un criterio tomado a posteriori -en el marco de los trabajos de limpieza y acondicionamiento- o si efectivamente se trata de asociaciones observadas durante la excavación.

\section{REFERENCIAS BIBLIOGRÁFICAS}

Alemán Aguilera, I., M. C. Botella López y L. Ruiz Rodríguez (2000). Determinación sexual mediante análisis discriminante del húmero. En L. Caro Dobón, H. Rodríguez Otero, E. Sánchez Compadre, B. López Martínez y M. J. Blanco Villegas (eds.), Tendencias Actuales de Investigación en la Antropología Física Española: 159-164. España, Universidad de León.

Ambrose, S. H. (1990). Preparation and characterization of bone and tooth collagen for isotopic analysis. Journal of Archaeological Science 17: 431-451.

Andrews, P. (1990). Owls, Caves and Fossils. Predation, Preservation and Accumulation of Small Mammal Bones in Caves, With an Analysis of the Pleistocene Cave Faunas from Westbury-Sub-Mendip, Somerset, U.K. Chicago, University of Chicago Press.

Aragón, E. y N. Franco (1997). Características de rocas para la talla por percusión y propiedades petrográficas. Anales del Instituto de la Patagonia (Serie Ciencias Humanas)25: 187-199.

Aschero, C. (1975). Ensayo para una clasificación morfológica de artefactos líticos aplicados a estudios tipológicos comparativos (Informe a CONICET). Ms.

Aschero, C. (1983). Ensayo para una clasificación morfológica de artefactos líticos aplicada a estudios tecnológicos comparativos. Apéndice A-C. Revisión para la cátedra de Ergología y Tecnología (FFyL, UBA). Ms. 


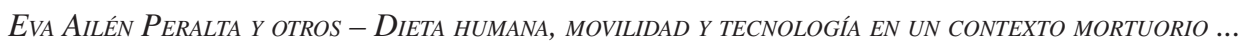

Aschero, C. y S. Hocsman (2004). Revisando cuestiones tipológicas en torno a la clasificación de artefactos bifaciales. En M. Ramos, A. Acosta y D. Loponte (eds.), Temas de Arqueología. Análisis Lítico: 7-25. Luján, Buenos Aires, Universidad Nacional de Luján.

Bárcena, R. (2001). Prehistoria del centro oeste argentino. En E. Berberian y A. Nielsen (eds.), Historia Argentina Prehispánica, volumen 2: 561-634. Córdoba, Editorial Brujas.

Bárcena, J. R., F. A. Roig, V. G. Roig (1985). Aportes arqueofito-zoológicos para la Prehistoria del NO de la provincia de Mendoza: La excavación de Agua de La Tinaja I. Trabajos de prehistoria 42: 311.

Barlow, K. R. (2002). Predicting maize agriculture among the Fremont: an economic comparison of farming and foraging in the American Southwest. American Antiquity 67: 65-88.

Behrensmeyer, A. K. (1978). Taphonomic and ecologic information from bone weathering. Paleobiology 4(2): 150-162.

Berrizbeitia, E. (1989). Sex determination with the head of the radius. Journal of Forensic Sciences 34(5): 1206-1213.

Bernal, V., S. D'Abramo, F. Gordón, P. N. González, y S. I. Pérez (2020). Mobility of human populations in the Curi Leuvú basin, Northwest Patagonia, during the Holocene: An approach based on oxygen isotopes. Journal of Archaeological Science: Reports 34, 102636.

Bernal, V., V.A. Cobos, S. I. Pérez y P. N. González (2016a). La estructura espacial del registro bioarqueológico de la provincia del Neuquén durante el Holoceno. En F. Gordon, R. Barberena y V. Bernal (eds.), El Poblamiento Humano del Norte de Neuquén. Estado Actual del Conocimiento y Perspectivas: 123-144. Buenos Aires, Ashpa.

Bernal, V., P. González, F. Gordón, S. I. Pérez (2016b). Exploring dietary patterns in the southernmost limit of prehispanic agriculture in America by using Bayesian stable isotope mixing models. Current Anthropology 57: 230-239.

Berón, M. (2007). Circulación de bienes como indicador de interacción entre las poblaciones de la Pampa occidental y sus vecinos. En C. Bayón, A. Pupio, M. I. González, N. Flegenheimer y M. Frére (eds.), Arqueología en las Pampas: 345-364. Buenos Aires, Sociedad Argentina de Antropología.

Berón, M., L. Prates y F. Prevosti (2015). Una historia de perros: Mitos y certezas sobre su origen y dispersión en América. Asociación Civil Ciencia Hoy 25(146): 39-45.

Binford, L. R. (1981). Bones. Ancient Men and Modern Myths. Londres, Academic press.

Binford, L. R. y W. J. Chasko (1976). Nunamiut demographic history: a provocative case. En B. Ezra y W. Zubrow (eds.), Demographic Anthropology: Quantitative Approaches: 63-143. Albuquerque, University of New Mexico.

Bocquet-Appel, J. P. (2002). The palaeoanthropological traces of Neolithic demographic transition. Current Anthropology 43: 638-650.

Bocquet-Appel, J. P. y J. Dubouloz (2003). Les traces paléoanthropologiques et archéologiques d'une transition démographique néolithique en Europe. Bulletin de la Societé Préhistorique Française 100(4): 699-714.

Bocquet-Appel, J. P. y J. Dubouloz (2004). Expected palaeoanthropological and aechaeological signal from a Neolithic demographic transition on a worldwide scale. Documenta Praehistorica 31: 25-33.

Bocherens, H. y D. G. Drucker (2003). Trophic level isotopic enrichment of carbon and nitrogen in bone 
Relaciones de la Sociedad Argentina de Antropología 46 (2), julio-diciembre 2021: 561-594

collagen: Case studies from recent and ancient terrestrial ecosystems. International Journal of Osteoarchaeology 13: 46-53.

Boeri, P., L. Piñuel, S. Sharry y D. Barrio (2017). Caracterización nutricional de la harina integral de algarroba (Prosopis alpataco) de la norpatagonia Argentina. Revista de la Facultad de Agronomía 116(1): 129-140.

Bruzek, J. (1995). Diagnose sexuelle á l'aide de l'analyse discriminante appliquée au tibia. Antropología Portuguesa 13: 93-106.

Bruzek, J. (2002). A method for visual determination of sex, using the human hip bones. American Journal of Physical Anthropology 117(2): 157-168.

Buikstra, J. E., L. W. Konigsberg y J. Bullington (1986). Fertility and the development of agriculture in the prehistoric Midwest. American Antiquity 51(3): 528-546.

Buikstra, J. E. y J. L. Mielke (1985). Demography, diet and health. En R. Gilbert y J. Mielke (eds.), The Analysis of Prehistoric Diets: 360-422. Orlando, Academic Press.

Buikstra, J. E. y D. H. Ubelaker (1994). Standards for Data Collection from Human Skeletal Remains. Fayetteville, Arkansas Archeological Survey Research Series 44.

Buzon, M. R., C. A. Conlee y G. J. Bowen (2011). Refining oxygen isotope analysis in the Nasca region of Peru: an investigation of water sources and archaeological samples. International Journal of Osteoarchaeology 21(4): 446-455.

Byrd, B. F., A. Cornellas, J. W. Eerkens, J. S. Rosenthal, T. R. Carpenter, A. Leventhal y J. A. Leonard (2013). The role of canids in ritual and domestic contexts: new ancient DNA insights from complex hunter-gatherer sites in prehistoric Central California. Journal of Archaeological Science 40(4): 2176-2189.

Cheung, C. y P. Szpak (2020). Interpreting past human diets using stable isotope mixing models. Journal of Archaeological Method and Theory, https://doi.org/10.1007/s10816-020-09492-5

Civalero, M. T. y N. V. Franco (2003). Early human occupations in Western Santa Cruz Province, Southermost South America. Quaternary International 109-110: 77-86.

Cohen, M. y G. Armelagos (1984). Paleopathology at the Origins of Agriculture. Nueva York, Academic Press Inc.

Coltrain, J. y S. Leavitt (2002). Climate and diet in Fremont prehistory: Economic variability and abandonment of maize agriculture in the Great Salt Lake Basin. American Antiquity 67: 453-485.

Corbat, M., A. F. Gil, R. Bettinger, G. A. Neme y A. Zangrando (2020). Ranking de recursos y dieta óptima en desiertos nordpatagónicos. Implicancias para el estudio de la subsistencia humana. Latin American Antiquity (en evaluación).

Cowal, L. S y R. F. Pastor (2008). Dimensional variation in the proximal ulna: evaluation of a metric method for sex assessment. American Journal of Physical Anthropology 135: 469-478.

Fernández, F. J., F. Ballejo, G. Moreira, E. Tonni, L. J. M. De Santis (2011). Roedores cricétidos de la provincia de Mendoza. Guía cráneo-dentaria orientada para su aplicación en estudios zooarqueológicos. Buenos Aires, Sociedad Argentina de Antropología-Universitas Sarmiento.

Fernández, F. J., C. I. Montalvo, Y. Fernández-Jalvo, P. Andrews y J. M. López (2017). A re-evaluation of the taphonomic methodology for the study of small mammal fossil assemblages of South America. Quaternary Science Reviews 155: 37-49. 
EVA Ailén Peralta y otros - Dieta humana, movilidad y tecnología en un Contexto mortuorio ...

Fernandes, R. (2016). A simple (r) model to predict the source of dietary carbon in individual consumers. Archaeometry 58(3): 500-512.

Fernandes, R., P. Grootes, M. J. Nadeau y O. Nehlich (2015). Quantitative diet reconstruction of a Neolithic population using a Bayesian mixing model (FRUITS): The case study of Ostorf(Germany). American Journal of Physical Anthropology 158(2): 325-340.

Fernandes, R., A. R. Millard, M. Brabec, M-J. Nadeau y P. Grootes (2014). Food Reconstruction Using Isotopic Transferred Signals (FRUITS): A bayesian model for diet reconstruction. PLoS ONE 9(2): e87436.

Gambier, M. (1977). La cultura de Ansilta. San Juan, Instituto de Investigaciones Arqueológicas y Museo, Facultad de Filosofía, Humanidades y Artes, Universidad Nacional de San Juan.

Gambier, M. (1980). Excavaciones en la Gruta de El Manzano, Río Grande-Mendoza. Boletín $N^{\circ}$ 1, Museo de Ciencias Naturales y Antropológicas "J. C. Moyana”.

Giardina, M. A., M. Corbat, C. Otaola, M. L. Salgán, A. Ugan, G. A. Neme y A. F. Gil (2014). Recursos y dietas humanas en Laguna Llancanelo (Mendoza; nordpatagonia): una discusión isotópica del registro arqueológico. Magallania 42: 111-131.

Giardina, M. A., M. Corbat, E. Peralta, G. Cochero, F. Franchetti y M. L. Salgán (2015). El registro arqueológico en sitio La Olla (San Rafael, Mendoza): implicaciones para las ocupaciones humanas en el valle medio del río Atuel. Revista del Museo de Antropología de Córdoba 8 (1): 51-66.

Gil, A. F. (1997-98). Cultígenos prehispánicos en el sur de Mendoza. Discusión en torno al límite meridional de la agricultura andina. Relaciones XXII-XXIII: 295-318.

Gil, A. F. (2003). Zea mays on the South American Periphery: Chronology and dietary importance. Current Anthropology 44 (2): 295-300.

Gil, A. F., M. A. Giardina, G. A. Neme y A. Ugan (2014). Demografía humana e incorporación de cultígenos en el centro occidente argentino: explorando tendencias en las fechas radiocarbónicas. Revista Española de Antropología Americana 44(2): 523-553.

Gil, A. F., L. P. Menéndez, J. P. Atencio, E. A. Peralta, G. A. Neme y A. Ugan (2018). Estrategias humanas, estabilidad y cambio en la frontera agrícola sur americana. Latin American Antiquity 29 (1): 6-26.

Gil, A. F. y G. A. Neme (2010). Registro arqueológico en la cuenca media del Atuel: viejos y nuevos problemas; viejos y nuevos datos. En M. Zárate, A. F. Gil y G. A. Neme (eds.), Condiciones Paleoambientales y ocupaciones humanas durante la transición Pleistoceno-Holoceno de Mendoza: 239-276. Buenos Aires, Sociedad Argentina de Antropología.

Gil, A. F., G. A. Neme y R. H. Tykot (2011). Stable isotopes and human diet in central western Argentina. Journal of Archaeological Science 38: 1395-1404.

Gil, A. F., G. A. Neme, A. Ugan y R. H. Tykot (2012). Isótopos estables $\left({ }^{13} \mathrm{C},{ }^{15} \mathrm{~N}\right.$ y $\left.{ }^{18} \mathrm{O}\right)$ en la arqueología del sur de Mendoza. En G. A. Neme y A. F. Gil (comps.), Paleoecología Humana en el Sur de Mendoza: Perspectivas Arqueológicas: 135-156. Buenos Aires, Sociedad Argentina de Antropología.

Gil, A. F., R. H. Tykot, G. A. Neme y N. Shelnut (2006). Maize on the frontier: isotopic and macrobotanical data from central-western Argentina. En Staller, R. H. Tykot y B. Benz (eds.), Histories of Maize: 199-214. Nueva York, Academic Press.

Gil, A. F., R. Villalba, F. R. Franchetti, C. Otaola, C. Abbona, E. A. Peralta y G. A. Neme (2020). Between 
Relaciones de la Sociedad Argentina de Antropología 46 (2), julio-diciembre 2021: 561-594

foragers and farmers: climate change and human strategies in Northwestern Patagonia. Quaternaryinternational 3(2): 17-37, DOI:10.3390/quat3020017.

González, M. E. (2014). Procesos de formación y efectos tafonómicos en entierros humanos: el caso del sitio Arroyo Seco 2 en la región pampeana, Argentina. Relaciones XXXIX (1): 175-202.

Guiry, E. (2012). Dogs as analogs in stable isotope-based human paleodietary reconstructions: a review and considerations for future use. Journal of Archaeological Method and Theory 19(3): 351-376.

Hedges, R. E. M., R. E. Stevens y P. L. Koch (2005). Isotopes in bones and teeth. En M. J. Leng (ed.), Isotopes in Palaeoenvironmental Research: 117-145. Países Bajos, Springer.

Hogg, A., T. J Heaton, Q. Hua, J. G Palmer, C. S. M. Turney, J. Southon, A. Bayliss, P. G Blackwell, G. Boswijk, C. Bronk Ramsey, C. Pearson, F. Petchey, P. Reimer, R. Reimer y L. Wacker (2020). SHCal20 Southern Hemisphere Calibration, 0-55,000 Years cal BP. Radiocarbon 62(4): 759-778.

Holland, T. (1991). Sex assessment using the proximal tibia. American Journal of Physical Anthropology 85: 221-227.

Iacumin, P., H. Bocherens, A. Mariotti y A. Longinelli (1996). Oxygen isotope analyses of co-existing carbonate and phosphate in biogenic apatite: a way to monitor diagenetic alteration of bone phosphate? Earth and Planetary Science Letters: 142-146.

Introna, F. Jr, G. Di Vella y C. P. Campobasso (1997). Sex determination by discriminant analysis of calcanei measurements. Journal of Forensic Sciences 42: 725-728.

Kochi, S. (2017). Paleodietas en cazadores-recolectores del canal Beagle durante el Holoceno tardío. Intersecciones en Antropología 18: 329-339.

Lagiglia, H. A. (1968). Secuencias culturales del Centro-Oeste argentino: valles del Atuel y Diamante. Revista Científica de Investigaciones 1(4): 159-174.

Lagiglia, H. A. (1982). El proceso de agriculturización en el sur de Cuyo: la cultura del Atuel II. Actas del V Congreso Nacional de Arqueología Argentina 2: 231-254. San Juan, Argentina.

Lagiglia, H. A. (1991). Acerca de los hallazgos indígenas de Cañada Seca. Boletín del Instituto de Ciencias Naturales 22: 3-4.

Lagiglia, H. A. (1999). Nuevos fechados radiocarbónicos para los agricultores incipientes del Atuel. Actas del XII Congreso Nacional de Arqueología Argentina 3: 239-250. La Plata, Argentina.

Lagiglia, H. A. (2001). Los orígenes de la agricultura en la Argentina. En E. Berberian y A. Nielsen. (eds.), Historia Argentina Prehispánica I: 41-81. Córdoba, Editorial Brujas.

Lagiglia, H. A. (2009). Indicadores arqueológicos de movilidad cultural en el centro-oeste argentino y aledaños. Sociedades de paisajes áridos y semiáridos 1: 15-43.

Lema, V. S., C. Della Negra y V. Bernal (2012). Explotación de recursos vegetales silvestres y domesticados en Neuquén: implicancias del hallazgo de restos de maíz y algarrobo en artefactos de molienda del Holoceno tardío. Magallania 40(1): 229-247.

Llano, C., P. Sosa; C. Sánchez Campo y R. Barberena (2019). Arqueobotánica de Cueva Huenul 1 (Neuquén, Argentina): selección y procesamiento de especies vegetales. Intersecciones en Antropología 20: 211-223.

López, J. M., G. Neme y A. F. Gil (2019). Resource intensification and zooarchaeological record in the southern 


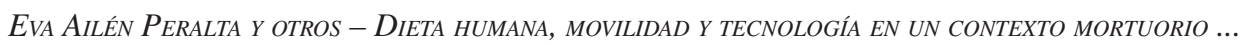

margins of pre-Hispanic Andean agriculture. Archaeological and Anthropological Sciences 11: 5287-5300.

López-Bueis, I., B. H. Robledo, P. Del Río y G. J. Trancho (2000). Identificación sexual del cúbito mediante funciones discriminantes. En L. Caro Dobón, H. Rodríguez Otero, E. Sánchez Compadre, B. López Martínez y M. J. Blanco Villegas (eds.), Tendencias Actuales de Investigación en la Antropología Física Española: 173-178. España, Universidad de León.

Lovejoy, C. O., R. S. Meindl, T. Pryzbeck y R. P. Mensforth (1985). Chronological methamorphosis of the auricular surface of the ilium: a new method for the determination of adult skeletal age at death. American Journal of Physical Anthropology 68: 15-28.

Luna, L. H. (2008). Estructura Demográfica, Estilo de Vida y Relaciones Biológicas de Cazadores Recolectores en un Ambiente de Desierto. Sitio Chenque I (Parque Nacional Lihué Calel, provincia de La Pampa). Oxford, BAR International Series 1886, Archaeopress.

Luz, B., Y. Kolodny y M. Horowitz (198 4). Fractionation of oxygen isotopes between mammalian bonephosphate and environmental drinking water. Geochimica et Cosmochimica Acta 48(8): 1689-1693.

Lyman, R. L. (1994). Vertebrate Taphonomy. Cambridge, Cambridge University Press.

Meindl R. S. y C. O. Lovejoy (1985). Ectocranial suture closure: a revised method for the determination of skeletal age at death based on the lateral-anterior sutures. American Journal of Physical Anthropology 68: 57-66.

Meindl, R. S. y C. O. Lovejoy (1989). Age changes in the pelvis: implications for paleodemography. En M. Yasar Isçan (ed.), Age Markers in the Human Skeleton: 137-168. Springfield, Illinois, Charles C. Thomas Publisher.

Meindl, R. S., C. O. Lovejoy y R. P. Mensforth (1980). Multifactorial determination of skeletal population of known age. American Journal of Physical Anthropology 52: 255.

Mengoni Goñalons, G. (1988). Análisis de materiales faunísticos arqueológicos. Xama 1: 71-120.

Mengoni Goñalons, G. (1999). Cazadores de Guanacos de la Estepa Patagónica. Buenos Aires, Sociedad Argentina de Antropología.

Mondini, M. (2003). Formación del registro arqueofaunístico en abrigos rocosos de la Puna argentina. Tafonomía de carnívoros. Tesis Doctoral inédita, Facultad de Filosofía y Letras, Universidad de Buenos Aires.

Moore, J. W. y B. X. Semmens (2008). Incorporating uncertainty and prior information into stable isotope mixing models. Ecology Letters 11: 470-480.

Morrison, D. J., B. Dodson, C. Slatery T. Preston (2000). ${ }^{13}$ C natural abundance in the British diet: implications for ${ }^{13} \mathrm{C}$ breath tests. Rapid Communications in Mass Spectrometry 14(15): 1321-1324.

Murphy, A. M. (2002). The calcaneus: sex assessment of prehistoric New Zealand Polynesian skeletal remains. Forensic Science International 129: 205-208.

Nakamura, K., D. A. Schoeller, F. J. Winkler y H. L. Schmidt (1982). Geographical variations in the carbon isotope composition of the diet and hair in contemporary man. Biological Mass Spectrometry 9(9): 390-394.

Neme, G. A. y A. F. Gil (2002). La explotación faunística y la frecuencia de partes esqueletarias en el registro arqueológico del sur mendocino. En A. F. Gil y G. A. Neme (eds.), Entre Montañas y Desiertos: Arqueología del Sur de Mendoza: 141-155. Buenos Aires, Sociedad Argentina de Antropología.

Neme, G. A. y A. F. Gil (2005). Aportes para la discusión del intercambio en el sur de Mendoza. Actas del XVI Congreso Nacional de Arqueología Chilena: 317-326. Concepción, Chile. 
Neme, G. A. y A. F. Gil (2012). El registro arqueológico del sur de Mendoza en perspectiva biogeográfica. En G. A. Neme y A. F. Gil (comps.), Paleoecología Humana en el Sur de Mendoza: Perspectivas Arqueológicas: 255-279. Buenos Aires, Sociedad Argentina de Antropología.

Novellino, P. S. (2002). Bioarqueología en el sur de Mendoza. En A. F. Gil y G. A. Neme (comps.), Entre Montañas y Desiertos: Arqueología del Sur de Mendoza: 119-139. Buenos Aires, Sociedad Argentina de Antropología.

Novellino, P. S. y A. F. Gil (2007). Estrés nutricional, hipoplasia y explotación de recursos en el centro sur de Mendoza (Argentina). Intersecciones en Antropología 8: 17-29.

Novellino, P. S., A. F. Gil, G. A. Neme y V. Durán (2004). El consumo de maíz en el Holoceno tardío del oeste argentino: isótopos estables y caries. Revista Española de Antropología Americana 34: 85-110.

Novellino, P. S. y R. A. Guichón (1997-98). Comparación de indicadores de dieta y salud entre el sur de Mendoza y el sur de San Juan-norte de Mendoza. Relaciones XXII-XXIII: 125-138.

Otaola, C., M. A. Giardina, M. Corbat y F. J. Fernández (2012). Zooarqueología en el sur de Mendoza: integrando perspectivas en un marco biogeográfico. En G. A. Neme y A. F. Gil (comps.), Paleoecología Humana en el Sur de Mendoza: Perspectivas Arqueológicas: 85-116. Buenos Aires, Sociedad Argentina de Antropología.

Otaola, C., A. Ugan y A. F. Gil (2018). Environmental diversity and stable isotope variation in faunas: Implications for human diet reconstruction in Argentine mid-latitude desert. Journal of Archaeological Science 20: 57-71.

Parnell, A. C., D. L. Phillips, S. Bearhop, B. X. Semmens, E. J. Ward, J. W. Moore, A. L. Jackson, J. Grey, D. J. Kelly y R. Inger (2013). Bayesian stable isotope mixing models. Environmetrics 24: 387-399.

Peralta, E. A. (2015). Subsistencia y movilidad en la cuenca media del Atuel en el Holoceno Tardío: Una perspectiva bioarqueológica, aplicando el análisis de marcadores de estrés ocupacional. Tesis de licenciatura inédita, Facultad de Filosofía y Letras, Universidad de Buenos Aires.

Peralta, E. A. (2017). Cambios y continuidades en la movilidad humana a finales del Holoceno tardío: cambios entésicos, lesiones articulares e isótopos estables en el sur de Mendoza. Revista del Museo de Antropología 10(2): 157-166.

Peralta, E. A. (2019). Demografía humana, dieta y actividad en los límites de la dispersión agrícola: tendencias bioarqueológicas en el sur de Mendoza a finales del Holoceno tardío. Tesis doctoral inédita, Facultad de Filosofía y Letras, Universidad de Buenos Aires.

Peralta, E. A. (2020). Tendencias en el uso del cuerpo en el valle del Atuel (sur de Mendoza) durante el Holoceno tardío final. InterSecciones en Antropología 21(2): 187-200.

Phillips, D. L. (2012). Converting isotope values to diet composition: the use of mixing models. Journal of Mammalogy 93(2): 342-352.

Phillips, D. L., R. Inger, S. Bearhop, A. L. Jackson, J. W. Moore, A. C. Parnell, B. X. Semmens y E. J. Ward (2014). Best practices for use of stable isotope mixing models in foodweb studies. Canadian Journal of Zoology 92: 823-835.

Pía, M. V., A. J. Novaro, M. Lucherini, J. I. Reppucci y A. E. J. Valenzuela (2019). Lycalopex culpaeus. En SAyDS-SAREM (eds.), Categorización 2019 de los mamíferos de Argentina según su riesgo de extinción. Lista Roja de los mamíferos de Argentina. [En línea] [Consultado el 10 de diciembre de 2020] Disponible en: http://cma.sarem.org.ar 


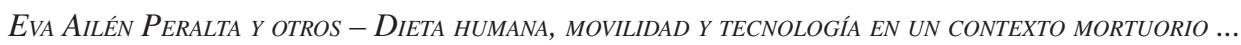

Popović, D., V. Mendoza España, M.Ziółkowski, P.Weglenski y M. Baca(2020). Molecular species assignment and dating of putative pre-Columbian dog remains excavated from Bolivia. Journal of Archaeological Science Reports 31, 102273

Prates, L. (2014). Crossing the boundary between humans and animals: the extinct fox Dusicyon avus from a hunter-gatherer mortuary context in Patagonia (Argentina). Antiquity 88: 1201-1212.

Prevosti, F. J., F. Santiago, L. Prates y M. Salemme (2011). Constraining the time of extinction of the South American fox Dusicyon avus (Carnivora, Canidae) during the late Holocene. Quaternary International 245: 209-17. http://dx.doi.org/10.1016/j.quaint.2011.02.010

Prevosti, F. J., V. Segura, G. Cassini y G. M. Martín (2013). Revision of the systematic status of patagonian and pampean gray foxes (Canidae: Lycalopex griseus and L. gymnocercus) using $3 \mathrm{~d}$ geometric morphometrics. Mastozoología Neotropical 20: 289-300.

Prevosti, F. J., M. Ubilla y D. Perea (2009). Large extinct canids from the Pleistocene of Uruguay: systematic, biogeographic and palaeoecological remarks. Historical Biology 21(1-2): 79-89.

Ríos Frutos, L. (2003). Brief communication: sex determination accuracy of the minimum supero-inferior femoral neck diameter in a contemporary rural Guatemalan population. American Journal of Physical Anthropology 122: 123-126.

Robledo, B. H., I. López-Bueis, J. A. Sánchez y G. J. Trancho (2000). Peroné: funciones discriminantes para la determinación sexual. En L. Caro Dobón, H. Rodríguez Otero, E. Sánchez Compadre, B. López Martínez y M. J. Blanco Villegas (eds.), Tendencias Actuales de Investigación en la Antropología Física Española: 179-186. España, Universidad de León.

Roig, V. G. (1965). Elenco sistemático de los mamíferos y aves de la provincia de Mendoza y notas sobre su distribución geográfica. Boletín de estudios geográficos 12(49): 175-222.

Salgán, M. L. (2015). Disponibilidad, estrategias de aprovisionamiento y uso de recursos líticos en La Payunia, sur de Mendoza. Revista del Museo de Antropología 8(2): 119-132.

Samec, C. T., M. Pirola y V. Killian Galván (2019). Lineamientos para la publicación de resultados isotópicos en antropología biológica y arqueología. Revista Argentina de Antropología Biológica 21(2): 007.

Sugrañes, N., M. P. Pompei, G. A. Neme y A. F. Gil (2020). Tecnología y movilidad en el Atuel Medio: el registro arqueológico del sitio Los Gallegos 1 (San Rafael, Mendoza). Comechingonia. Revista de Arqueología 25(2): 5-36.

Scheuer, L. y S. Black (2000). Developmental Juvenile Osteology. Londres, Academic Press.

Seidemann, R., C. M. Stojanowsky y G. H. Doran (1998). The use of the superior-inferior femoral neck diameter as a sex assessor. American Journal of Physical Anthropology 107: 305-313.

Sharp, Z. (2007). Principles of Stable Isotope Geochemistry. New Jersey, Prentice Hall, Upper Saddle River.

Silva, A. M. (1995). Sex assessment using the calcaneus and talus. Antropología Portuguesa 13: 107-119.

Smith, B. D. (2001). Low-level food production. Journal of Archaeological Research 9: 1-43.

Steele, D. G. (1976). The estimation of sex on the basis of the talus and calcaneus. American Journal of Physical Anthropology 45: 581-588.

Szpak, P., C. D. White, F. J. Longstaffe, J. F. Millaire y V. F. Vásquez Sánchez (2013). Carbon and Nitrogen 
Relaciones de la Sociedad Argentina de Antropología 46 (2), julio-diciembre 2021: 561-594

isotopic survey of northern peruvian plants: baselines for paleodietary and paleoecological studies. PLoS ONE 8(1): 753-763.

Todd, T. W. (1921a). Age changes in the pubic bone. I: the male white pubis. American Journal of Physical Anthropology 3: 285-334.

Todd, T. W. (1921b). Age changes in the pubic bone. III: the pubis of the white female. IV: the pubis of the female white-negro hybrid. American Journal of Physical Anthropology 4: 1-70.

Todd, L. C. y D. J. Rapson (1988). Long bone fragmentation and interpretation of faunal assemblages: approaches to comparative analysis. Journal of Archaeological Science 15: 307-325.

Trancho, G. J., I. López-Bueis, B. H. Robledo y J. A. Sánchez (2000). Diagnóstico sexual del radio mediante funciones discriminantes. En L. Caro Dobón, H. Rodríguez Otero, E. Sánchez Compadre, B. López Martínez y M. J. Blanco Villegas (eds.), Tendencias Actuales de Investigación en la Antropología Física Española: 165-172. España, Universidad de León.

Trancho, G., B. H. Robledo, I. López-Bueis y J. A. Sánchez (1997). Sexual determination of the femur using discriminant functions. Analysis of a Spanish population of known sex and age. Journal of Forensic Sciences 42(2): 181-185.

Tykot, R. H., F. Falabella, M. T. Planella, E. Aspillaga, L. Sanhueza y C. Becker (2009). Stable isotopes and archaeology in central Chile: Methodological insights and interpretative problems for dietary reconstruction. International Journal of Osteoarchaeology 19: 156-170.

Ugan, A., G. A. Neme, A. F. Gil, J. Coltrain, R. H. Tykot y P. S. Novellino (2012). Geographic variation in bone carbonate and water $\delta^{18} \mathrm{O}$ values in Mendoza, Argentina and their relationship to prehistoric economy and settlement. Journal of Archaeological Science 39: 2752-2763.

United States Department of Agriculture (USDA) (2015). USDA National Nutrient Database for Standard Reference, Release 28. [En línea] [Consultado el 10 de diciembre de 2020] Disponible en: http://ndb.nal. usda.gov/ndb/search/list.

Urbistondo, J. y J. Pereyra (2015). Caracterización del Valor Nutricional de Alimentos. Montevideo, Buenos Aires, PROCISUR, IICA.

White, T. y P. Folkens (2005). The Human Bones Manual. Londres, Elsevier Academic Press.

Wilbur, A. K. (1998). The utility of hand and foot bones for the determination of sex and the estimation of stature in a prehistoric population from West-Central Illinois. International Journal of Osteoarchaeology 8: 180-191.

Winterhalder, B. y C. Goland (1997). An evolutionary ecology perspective on diet choice, risk, and plant domestication. En K. Gremillion (ed.), Peoples, Plants, and Landscapes: Studies in Paleoethnobotany: 23160. Tuscaloosa, University of Alabama Press.

Winterhalder, B. y D. J. Kennett (2006). Behavioral Ecology and the Transition to Agriculture. Berkeley, University of California Press.

Winterhalder, B. y D. J. Kennett (2020). Seven behavioral ecology reasons for the persistence of foragers with cultivars. En M. Delacorte y J. L. Terry (eds.), Cowboy Ecologist: Essays in Honor of Robert L. Bettinger: 93-110. California, CARD (Center for Archaeological Research at Davis). 